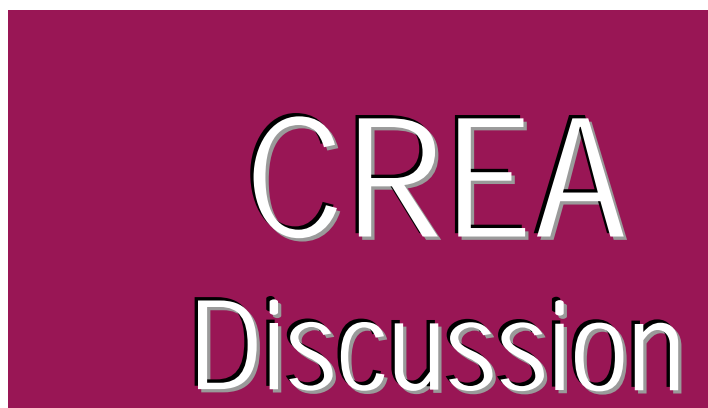
Paper

\title{
2014-06
}

Center for Research in Economic Analysis

University of Luxembourg

\section{Strategic Exploitation of a Common-Property Resource under Rational Learning about its Reproduction}

available online : http://wwwfr.uni.Iu/recherche/fdef/crea/publications2/discussion_papers

Christos Koulovatianos, CREA, Université du Luxembourg

March, 2013

For editorial correspondence, please contact: crea@uni.lu

University of Luxembourg

Faculty of Law, Economics and Finance

162A, avenue de la Faïencerie

L-1511 Luxembourg 


\title{
Strategic Exploitation of a Common-Property Resource Under Rational Learning About its Reproduction
}

\author{
Christos Koulovatianos* \\ University of Luxembourg, CREA, and CFS
}

March 21, 2014

* I thank the Editor, Georges Zaccour, and three anonymous referees, whose comments greatly improved this paper. I also thank Johannes Hörner, Ramon Marimon, Marc Santugini, and Daniel Seidmann for useful discussions and suggestions. An earlier version of this paper has been circulated in 2010, under the title "A Paradox of Environmental Awareness Campaigns", University Library of Munich, MPRA Paper 27260.

Corresponding address: Department of Economics, University of Luxembourg, 162A avenue de la Faïencerie, BRC 1.06E, L-1511 Luxembourg, email: christos.koulovatianos@uni.lu, Phone: +352-46-66-44-6356, Fax: +352-46-66-44-6341. 


\title{
Strategic Exploitation of a Common-Property Resource Under Rational Learning About its Reproduction
}

\begin{abstract}
We build a workable game of common-property resource extraction under rational Bayesian learning about the reproduction prospects of a resource. We focus on Markov-perfect strategies under truthful revelation of beliefs. For reasonable initial conditions, exogenously shifting the prior beliefs of one player towards more pessimism about the potential of natural resources to reproduce, can create anti-conservation incentives. The single player whose beliefs have been shifted towards more pessimism exhibits higher exploitation rate than before. In response, all other players reduce their exploitation rates in order to conserve the resource. However, the overall conservation incentive is weak, making the aggregate exploitation rate higher than before the pessimistic shift in beliefs of that single player. Due to this weakness in strategic conservation responses, if the number of players is relatively small, then in cases with common priors, jointly shifting all players' beliefs towards more pessimism exacerbates the commons problem.
\end{abstract}

Keywords: renewable resources, resource exploitation, non-cooperative dynamic games, Bayesian learning, stochastic games, commons, rational learning, uncertainty, beliefs JEL classification: D83, D84, C72, C73, O13, Q20, Q50, L70 


\section{Introduction}

Markets invest vast amounts in renewable natural resources. Yet, it is difficult to argue that investors have rational expectations about nature's stochastic law of resource renewal. In particular, a vivid debate among experts shows that important magnitudes regarding the fundamentals of natural-resource reproduction are obscure to both experts and the public. For example, the book by Lomborg (2001) scrutinizes this obscurity of experts' perceptions regarding shifts in nature's fundamentals due to "global warming". ${ }^{1}$ It appears that our understanding of natural-resource-market regulation can be enhanced by explicitly incorporating this documented obscurity in resource-extraction models. To this end, a plausible approach is to introduce (virtual) Bayesian econometricians who cope with model uncertainty in models of noncooperative resource extraction. Yet, even along this standardized modeling route, little is known about how extraction strategies are qualitatively affected by optimism/pessimism regarding resource-renewal prospects. Towards filling this theoretical gap, here we build an example, a workable resource-extraction model which shows the explicit dependence of strategic extraction mechanics on such belief biases and belief heterogeneity. ${ }^{2}$

\footnotetext{
1 Debates about acid rain (see, for example, Lomborg (2001, pp. 178-181)), or about a large-scale biodiversity deterioration (Lomborg (2001, pp. 249-257)) are related to shifts in fundamentals regarding the natural ability of resources to reproduce. While environmental changes are not disputed among experts as facts, the magnitude of such changes is a vivid topic of disagreement among experts. For example, the article by Rörsch et al. (2005) documents the opposition against Lomborg's (2001) book by a part of the scientific community. What we keep from this debate among experts for the purposes of this study is that investor uncertainty about environmental fundamentals is a plausible working hypothesis for analyzing investments in natural resources.

2 Despite that we analyze a specific example, the stochastic structure of our Bayesian-learning setup is general, i.e. it is not restricted by conjugate-priors assumptions, as it will be clearer in the model's outline below. This generality allows for a wide range of applications, extending even to the analysis of jump processes. This versatility of the example's stochastic structure may stimulate further theoretical and empirical investigation, not only because of the theoretical ideas which are demonstrated here, but also because the example may be fitted to actual data itself. Here, we restrict our analysis to demonstrating the example's mechanics theoretically.
} 
Our model is a game of common-property resource exploitation. Players are unaware of nature's true parameters driving the ability of the resource to reproduce. So, players have initial beliefs about particular parameters behind nature's fundamentals, and collect data in each period in order to update these beliefs, using Bayes' rule. ${ }^{3}$ Belief heterogeneity is allowed in our model, and its presence is justified by the fact that different players may start with different initial understanding of fundamentals through available data. For example, if players are countries, then it is not unreasonable to think that cross-country differences in schooling levels may trigger differences in interpretations of existing evidence, such as global-warming data, biodiversity trends and their role in a particular resource's reproduction potential, etc. ${ }^{4}$ In practice, such cross-country differences in beliefs can be measured through surveys. In turn, belief biases and their heterogeneity across players are taken into account by other players and affect noncooperative strategic resource extraction.

It is desirable to have a clear interplay between beliefs and the model's fundamentals. To this end we employ the concept of rational Bayesian learning under truthful revelation of beliefs. Rational-learning investors envisage the arrival of new information and anticipate learning in the future. The distinct feature of rational learning is that the mapping of priors to posteriors implied by Bayes' rule is incorporated in the recursive problem of each player together with other recursions governing laws of motion of the problem's state variables. In other words, when learning is rational, prior beliefs become part of the problem's state

3 For example, collecting and processing data on each winter's temperature informs scientists and investors in natural-resource markets about the validity of their prior beliefs regarding a structural break due to "global warming".

4 This heterogeneity in prior beliefs is justified by differences in non-informative priors, which are priors formed before any data are available. Ad-hoc differences in non-informative priors can persist and can play a crucial role in the transitional dynamics of a game. For an introduction to non-informative priors and references on alternative criteria for choosing non-informative priors see, for example, Gelman et al. (2004, pp. 61-66). 
variables. ${ }^{5}$ Moreover, we focus on Markov-perfect Nash-equilibrium strategies. ${ }^{6}$ Our focus on this equilibrium concept enables us to use dynamic-programming techniques in order to tackle the problem. ${ }^{7}$

In order to obtain robust analytical solutions we combine the analytical virtues of two workable models. The first is the Levhari and Mirman (1980) example about strategic exploitation of renewable resources. The second is the framework by Koulovatianos, Mirman, and Santugini (2009) which combines dynamic programming with rational learning and provides analytical solutions. Similarly to the latter study, the stochastic structure of our model is general, i.e., our results do not depend on any specific assumptions about the densities of any random variables in our model. ${ }^{8}$

We obtain a unique and robust equilibrium in pure Markov-perfect strategies under truthful revelation of beliefs among all players. We show that if the priors of at least one player about the renewal prospects of the resource are exogenously shifted to being more

5 Our setup of Bayesian learners who anticipate learning in the future is similar to the case of rational learning examined by Guidolin and Timmermann (2007), Cogley and Sargent (2008), and Koulovatianos, Mirman, and Santugini (2009).

6 Focusing on Markov-perfect-Nash-equilibrium strategies may seem restrictive. Yet, even this simplified equilibrium concept involves considerable technical complexities. It was several years after the seminal example by Levhari and Mirman (1980) that Sundaram (1989) proved equilibrium existence for the more general deterministic version. Dutta and Sundaram (1992) were the first to extend analysis to the stochastic version of the game under rational expectations, while Amir (1996) contributed a remarkably general characterization of such stochastic games offering existence and uniqueness results. For a general survey of natural-resource games see Long (2011).

7 Kalai and Lehrer (1993) show a key result related to our rational-learning formulation. When Bayesian updating is envisaged by each player, then Bayesian updating of collected information will lead in the long run to accurate prediction of the future play of the game and rational expectations as a limit of behavior with probability one, as time goes to infinity. This consideration on the side of players, that learning will be completed in the long run, and its impact on strategies, is a key distinctive feature of rational learning from other forms of learning. (The way to make players envisage Bayesian updating in our setting that focuses on Markov-perfect Nash strategies, is to incorporate Bayes' rule in the Bellman equation of each player.) In their survey paper, Blume and Easley (1993) explain this distinction as well.

8 A recent paper that also combines strategic interaction extending the Levhari and Mirman (1980) model with rational Bayesian learning in a similar fashion to Koulovatianos, Mirman, and Santugini (2009) is Mirman and Santugini (2013). A key difference of the present paper from Mirman and Santugini (2013) is that here heterogeneous beliefs are allowed and the impact of belief changes of one player can be characterized using a general stochastic structure for learning. 
pessimistic than before, the response strategies of each player change. In particular, shifting one player's priors towards pessimism makes this particular player increase her exploitation rate. All other players' response to this shift in beliefs of one player is an effort to conserve the resource: each of the other players reduces her own individual extraction rate. However, these strategic conservation responses and incentives are not strong enough. Making at least one player more pessimistic than before always implies higher aggregate resource exploitation rates in the model.

The weakness of conservation incentives uncovered by our setup is crucial for understanding how pessimism affects the intensity of the commons problem. The commons-problem intensity is captured by the magnitude by which aggregate extraction rates increase as another player is added. If priors are the same across players, then, according to our model, the increase in aggregate exploitation rates due to adding another player is higher under more pessimistic identical beliefs across players only if the number of players is "small". This result is intuitive. A large number of players tends to partly conserve the resource in order to reduce the already high exploitation rate due to increases in pessimism by other players.

The model and much of the analysis of this paper first appeared in a previous version, Koulovatianos (2010). Another study using the framework developed in Koulovatianos (2010) is Agbo (2013). ${ }^{9}$ Agbo (2013) follows the analytical approach in Koulovatianos, Mirman, and Santugini (2009), which uses adaptive learning as an interim equilibrium concept standing between rational expectations and rational learning, in order to identify the so called "learning-anticipation effect". The "learning-anticipation effect" can be identified by comparing the adaptive-learning equilibrium with the rational-learning equilibrium, stressing

9 The proof regarding the solution of the linear system of multiple Nash-equilibrium necessary conditions, which appears as Lemma 3 in the Appendix of this paper, and which relies on the "matrix determinant lemma", first appeared in Koulovatianos (2010, pp. 27-29), and enabled the identification of the solution in both Koulovatianos (2010) and in Agbo (2013). 
how the knowledge that all players' values will be affected by future learning affects strategic behavior. The complementary contribution of this paper in relation to Agbo (2013) and Koulovatianos, Mirman, and Santugini (2009) is twofold. First, it introduces an alternative method of proof, which does not rely on a guess about the form of the value function in the infinite-horizon problem. Instead, it focuses on solving finite-horizon games and extending the solution to infinite horizon, giving the opportunity to verify the uniqueness of the Markov-perfect solution, under the assumption that each player's individual optimization

problem is always well-defined. Second, in the present study we emphasize how the interpretation of optimism/pessimism changes depending on the value of the resource stock in this model and we identify, (i) parametric constraints, and (ii) constraints on initial conditions, that help in keeping these interpretations consistent. In addition, here we study how beliefs affect the intensity of the commons problem under the assumption of identical priors across players, discussing how such insights can prove useful in prescribing environmental policies.

In Section 2 we present the model and its solution. In Section 3 we characterize the solution, providing results about how exploitation strategies respond to shifts in belief priors, and we analyze how beliefs affect the intensity of the commons problem. In Section 4 we make concluding remarks.

\section{Model}

Time is discrete and the horizon is infinite $(t \in\{0,1, \ldots\})$. There is a renewable resource, the stock of which is denoted by $k$. At time $t=0$, the stock is $k_{0}>0$.

\subsection{Nature's Law of Motion without Exploitation}

When nobody exploits the resource, the law of motion of the resource's stock is,

$$
k_{t+1}=B k_{t}^{\eta_{t}}, \quad t=0,1, \ldots,
$$


in which $B>0$ and parameter $\eta_{t} \in(0,1)$ is a serially uncorrelated random variable with time-invariant density function $\phi\left(\eta \mid \theta^{*}\right)$ and support $\mathcal{H} \subseteq(0,1)$, while $\theta^{*}$ is a vector of parameters which is constant over time and known by nature.

\subsection{Players, Payoff Functions, and Resource Exploitation}

Let a fixed set of $N \geq 1$ players, each having equal rights to exploiting the stock $k$, as the resource is perfectly rivalrous and nonexcludable. Each player $i \in\{1, \ldots, N\}$ consumes $c_{i, t}$ units of the stock $k$ in period $t \in\{0,1, \ldots\}$, before nature reveals its realization $\eta_{t}$ within period $t .^{10}$ So, after exploitation the law of motion becomes,

$$
k_{t+1}=B \cdot\left(k_{t}-\sum_{i=1}^{N} c_{i, t}\right)^{\eta_{t}}, \quad t=0,1, \ldots
$$

All players are infinitely-lived and have the same objective function, maximizing expected life-time utility from consumption. For player $i \in\{1, \ldots, N\}$ lifetime utility given by,

$$
E_{0}\left[\sum_{t=0}^{\infty} \delta^{t} \ln \left(c_{i, t}\right)\right]
$$

in which $\delta \in(0,1)$ is the discount factor. All players know the density function $\phi(\eta \mid \cdot)$, but do not know the true parameter vector $\theta^{*}$. In period 0 each player $i \in\{1, \ldots, N\}$ has a prior density function of beliefs about vector $\theta$ given by $\xi_{0}^{i}$. All players are Bayesian learners. So, given $\xi_{0}^{i}$, for any sequence of realizations of the shock $\eta,\left\{\eta_{t}\right\}_{t=0}^{\infty}$, the sequence $\left\{\xi_{t}^{i}\right\}_{t=0}^{\infty}$ is generated by,

$$
\xi_{t+1}^{i}\left(\theta \mid \eta_{t}\right)=\frac{\phi\left(\eta_{t} \mid \theta\right) \xi_{t}^{i}(\theta)}{\int_{\Theta} \phi\left(\eta_{t} \mid x\right) \xi_{t}^{i}(x) \mathrm{d} x}, t \in\{0,1, \ldots\} \text { and } i \in\{1, \ldots, N\},
$$

in which $\Theta$ is the time-invariant support of vector $\theta$.

10We use a player's index $i \in\{1, \ldots, N\}$ as subscript for variables and as superscripts for functions, and we drop it whenever it is redundant. 


\subsection{Assumptions about Players' Information}

For notational simplicity let $\Xi_{t} \equiv\left(\xi_{t}^{1}, \ldots, \xi_{t}^{N}\right)$. In period $t=0$, assume that all players know $\Xi_{0}$. Given the demanding technical nature of dynamic resource games with heterogeneous beliefs, this assumption offers convenience. Nevertheless, the assumption that each player knows the priors of all other players, is also reasonable for some fundamental applications. For example, if each player is a country that wishes to manage its fishing industry, it is reasonable to assume that each country is accurately informed about collective beliefs in other countries. Such information can be obtained through reading the press or through public-opinion-poll studies about environmental issues in foreign countries. In addition, it is reasonable to observe belief heterogeneity about environmental issues across different countries. For example, environmental-awareness campaigns have different intensity or importance within the list of alternative public-awareness issues in different countries.

Another key assumption is that players fully observe $\eta_{t}$ in all periods $t \in\{0,1, \ldots\}$. Assuming that additional noise is implicit in an observed signal does not add insights to understanding the effect of beliefs on players' strategies in our application.

\subsection{Equilibrium Concept under Rational Learning}

Definition 1 gives the central equilibrium concept we use throughout the paper.

Definition 1 A Rational-Learning Markov-Perfect Nash Equilibrium (RLMPNE) is a set of strategies of the form $\left\{c_{i}=C^{i}(k ; \Xi)\right\}_{i=1}^{N}$ such that, for all $i \in\{1, \ldots, N\}$, there exists a well-defined value function $V^{i}(k ; \Xi)$, satisfying the transversality condition,

$$
\lim _{t \rightarrow \infty} \delta^{t} E_{t}\left[\frac{\partial h^{i}\left(k_{t}^{*}, k_{t+1}^{*} ; \Xi_{t}\right)}{\partial k_{t}} k_{t}^{*}\right]=0
$$


throughout the equilibrium path $\left\{k_{t}^{*}\right\}_{t=0}^{\infty}$, in which,

$$
h^{i}\left(k_{t}, k_{t+1} ; \Xi_{t}\right) \equiv \ln \left[k_{t}-\sum_{j \neq i} C^{j}\left(k_{t} ; \Xi_{t}\right)-\left(\frac{k_{t+1}}{B}\right)^{\frac{1}{\eta_{t}}}\right],
$$

and $c_{i}=C^{i}(k ; \Xi)$ solves,

$$
\begin{aligned}
& V^{i}(k ; \Xi)=\max _{c_{i} \geq 0}\left\{\ln \left(c_{i}\right)+\right. \\
& \left.\quad+\delta \int_{\mathcal{H}} V^{i}\left(B \cdot\left[k-c_{i}-\sum_{j \neq i} C^{j}(k ; \Xi)\right]^{\eta} ; \hat{\Xi}(\cdot \mid \eta)\right)\left[\int_{\Theta} \phi(\eta \mid \theta) \xi^{i}(\theta) \mathrm{d} \theta\right] \mathrm{d} \eta\right\}
\end{aligned}
$$

subject to,

$$
\hat{\xi}^{i}(\theta \mid \eta)=\frac{\phi(\eta \mid \theta) \xi^{i}(\theta)}{\int_{\Theta} \phi(\eta \mid x) \xi^{i}(x) \mathrm{d} x}, \quad i=1, \ldots, N,
$$

in which $\Xi$ is common knowledge to all players $i \in\{1, \ldots, N\}$.

Notice that $V^{i}$ is the value function of player $i$. Despite the symmetry in all fundamentals of the game across players, function $V^{i}$ is distinguished from any other player's value function due to the asymmetry in beliefs. Initial beliefs, $\xi_{0}^{i}$, generally differ across players, and their distinctive role shown in the Bellman equation (5) of any player $i \in\{1, \ldots, N\}$, necessitates the distinction of $V^{i}$ from value functions of other players. Notice also that we have used a hat for denoting variables one period ahead.

\subsection{Rational-Learning Equilibrium}

Proposition 1 formally states the solution to the model under rational learning, which is the key result of this paper. For notational compactness we let function $\mu$ be,

$$
\mu(\theta) \equiv \int_{\mathcal{H}} \eta \phi(\eta \mid \theta) \mathrm{d} \eta,
$$

which captures the parameter-driven conditional expectation about nature's efficiency concerning natural resource reproduction in each period. First, notice that $\mu(\theta) \in(0,1)$ for all 
$\theta \in \Theta$, due to our assumptions about $\mathcal{H}$. Second, the law of motion given by (2) implies that, if $k_{t}-\sum_{i=1}^{N} C^{i}\left(k_{t} ; \Xi\right)>1$, then the higher the level of $\mu(\theta)$, the higher the expected ability of the resource to reproduce. The comparison between $k_{t}-\sum_{i=1}^{N} C^{i}\left(k_{t} ; \Xi\right)$ and unity has implications for both the expected growth rate of $k$ and for the variance of the growth rate. Specifically, take natural logarithms on both sides of equation $(2)$, subtract $\ln \left(k_{t}\right)$, to obtain,

growth rate of $k$ in period $t \equiv \ln \left(k_{t+1}\right)-\ln \left(k_{t}\right)=$

$$
=\ln (B)-\ln \left(k_{t}\right)+\eta_{t} \cdot \ln \left[k_{t}-\sum_{i=1}^{N} C^{i}\left(k_{t} ; \Xi\right)\right]
$$

Integrating both sides of $(7)$ with respect to $\eta$ throughout the support $\mathcal{H}$,

expected growth rate of $k$ in period $t \equiv E_{t}\left[\ln \left(k_{t+1}\right)-\ln \left(k_{t}\right)\right]=$

$$
=\ln (B)-\ln \left(k_{t}\right)+\mu(\theta) \cdot \ln \left[k_{t}-\sum_{i=1}^{N} C^{i}\left(k_{t} ; \Xi\right)\right] .
$$

Equation (8) shows that increases in $\mu(\theta)$ increase the growth rate of $k$ only if $k_{t}-\sum_{i=1}^{N} C^{i}\left(k_{t} ; \Xi\right)>$ 1 , otherwise, increases in $\mu(\theta)$ decrease the growth rate of $k$. So, whether a change in beliefs according to which $\mu(\theta)$ is anticipated to be higher, is interpreted as optimism requires to secure that $k_{t}-\sum_{i=1}^{N} C^{i}\left(k_{t} ; \Xi\right)>1$. In addition, equation (8) shows that also the conditional variance of the growth rate of $k$ also increases (decreases) for higher levels of $k_{t}-\sum_{i=1}^{N} C^{i}\left(k_{t} ; \Xi\right)$ as long as $k_{t}-\sum_{i=1}^{N} C^{i}\left(k_{t} ; \Xi\right)>(<) 1$. Since the nature of the decision rules $C^{i}\left(k_{t} ; \Xi\right), i=1, \ldots, N$, is crucial for all comparative statics and all optimism/pessimism interpretations, we proceed to Proposition 1 , which identifies $C^{i}\left(k_{t} ; \Xi\right)$. 
Proposition 1 There exists a unique pure-strategy RLMPNE solving the problem described by Definition 1, with all players exhibiting constant exploitation rates at all times, i.e. $C^{i}(k ; \Xi)$ is of the form $C^{i}(k ; \Xi)=c^{i}(\Xi) \cdot k$ with

$$
c^{i}(\Xi)=\frac{\left(\int_{\Theta} \frac{\delta \mu(\theta)}{1-\delta \mu(\theta)} \xi^{i}(\theta) \mathrm{d} \theta\right)^{-1}}{1+\sum_{j=1}^{N}\left(\int_{\Theta} \frac{\delta \mu(\theta)}{1-\delta \mu(\theta)} \xi^{j}(\theta) \mathrm{d} \theta\right)^{-1}}, \quad i=1, \ldots N,
$$

while the value function is of the form,

$$
V^{i}(k ; \Xi)=\kappa^{i}(\Xi)+\left[\int_{\Theta} \frac{1}{1-\delta \mu(\theta)} \xi^{i}(\theta) \mathrm{d} \theta\right] \cdot \ln (k), \quad i=1, \ldots N,
$$

in which $\kappa^{i}(\Xi)$ solves,

$$
\kappa^{i}(\Xi)-\delta \int_{\mathcal{H}} \kappa^{i}(\Xi(\cdot \mid \eta))\left[\int_{\Theta} \phi(\eta \mid \theta) \xi^{i}(\theta) \mathrm{d} \theta\right] \mathrm{d} \eta=\alpha(\Xi),
$$

with

$$
\begin{aligned}
& \alpha(\Xi) \equiv \delta \ln (B)+\left[1+\int_{\Theta} \frac{\delta \mu(\theta)}{1-\delta \mu(\theta)} \xi^{i}(\theta) \mathrm{d} \theta\right] \times \\
& \times \ln \left[1+\sum_{j=1}^{N}\left(\int_{\Theta} \frac{\delta \mu(\theta)}{1-\delta \mu(\theta)} \xi^{j}(\theta) \mathrm{d} \theta\right)^{-1}\right]-\ln \left[\int_{\Theta} \frac{\delta \mu(\theta)}{1-\delta \mu(\theta)} \xi^{i}(\theta) \mathrm{d} \theta\right],
\end{aligned}
$$

provided that function $\phi(\cdot \mid \theta)$ and priors $\Xi$ guarantee a bounded solution to the difference equation (11), for $i=1, \ldots N$.

Proof See the Appendix.

The condition given by (11) is imposed in order to guarantee that all players' value functions are well-defined, as required by Definition 1. In applications using specific functional forms for $\phi(\cdot \mid \theta)$ and $\Xi$, the condition given by (11) can help in order to identify parametric constraints that guarantee the boundedness of $\kappa^{i}(\Xi), i=1, \ldots, N$. A survey of methods for 
verifying whether solutions to difference equations such as (11) are bounded can be found, e.g. in Mawhin (2009).

Assuming that boundedness of $\kappa^{i}(\Xi), i=1, \ldots, N$ is guaranteed, an attractive feature of the solution described in Proposition 1 is that there is only one pure-strategy RLMPNE. In addition, the closed form of equation (9) allows us to explicitly understand the impact of other players' beliefs on player $i$ 's exploitation strategy. Before moving to characterizing the equilibrium we briefly derive the rational-expectations equilibrium.

\subsection{Benchmark Rational-Expectations Equilibrium}

Learning is passive in this framework. Under certain assumptions on the stochastic structure of learning, as $t \rightarrow \infty$, all players learn the true parameter with certainty, in which case the game described in Definition 1 converges to rational expectations. Convergence to rational expectations is the most usual assumption made in the literature. Yet, there are setups in which Bayesian learning does not lead to infinite precision regarding parameters that are to be learned, such as the continuous-time setup of Bayesian-learning about the hazard rate of a jump process described in Koulovatianos and Wieland (2011). Even so, a rationalexpectations equilibrium serves as a yardstick in order to understand the extent of belief bias, so we define it below for our framework.

A Rational-Expectations Markov-Perfect Nash Equilibrium (REMPNE) is a set of strategies of the form $\left\{c_{i}=C^{R E, i}(k)\right\}_{i=1}^{N}$ such that for all $i \in\{1, \ldots, N\}, c_{i}=C^{R E, i}(k)$ solves the problem given in Definition 1 after substituting the generic rational expectations distribution for $\xi$ (denote by $\xi^{R E}$ ), given by, $\xi^{R E}(\theta)=1$, if $\theta=\theta^{*}$, and $\xi^{R E}(\theta)=0$, if $\theta \neq \theta^{*}$, with $\xi_{i}=\xi^{R E}$ for all $i \in\{1, \ldots, N\}$. The derivation of strategies $\left\{c_{i}=C^{R E, i}(k)\right\}_{i=1}^{N}$ is immediate from equations (9), after substituting the generic rational expectations distribution $\xi^{R E}$ for all players. Given this immediate proof, Corollary 1 only states the unique Markov-perfect 
equilibrium under rational expectations.

Corollary 1 There is a unique pure-strategy REMPNE solving the problem described by Definition 1 with $\xi_{i}=\xi^{R E}$ for all $i \in\{1, \ldots, N\}$, where $\left\{c_{i}=C^{R E, i}(k)\right\}_{i=1}^{N}$ is such that all players exhibit constant exploitation rates at all times, i.e. $C^{R E, i}(k)$ is of the form $C^{R E, i}(k)=c^{R E, i} \cdot k$ with

$$
c^{R E, i}=\frac{\left(\delta \mu\left(\theta^{*}\right)\right)^{-1}-1}{1+N \cdot\left[\left(\delta \mu\left(\theta^{*}\right)\right)^{-1}-1\right]}, \quad i=1, \ldots N .
$$

One of the features of REMPNE is that under rational expectations all players have common priors. In addition, these priors under rational expectations reflect confidence that the correct parameter of nature is known by all players. Finally, it is straighforward to verify that, under rational expectations, the common-across players value function is always bounded with $\kappa\left(\Xi^{R E}\right)=\alpha\left(\Xi^{R E}\right) /(1-\delta)$, with $\kappa\left(\Xi^{R E}\right)$ and $\alpha\left(\Xi^{R E}\right)$ given by (11) and (12), calculated for the degenerate distribution $\Xi^{R E}$.

\section{Belief Bias, Resource Exploitation Rates, and the Commons Problem}

In this section we explore the impact of shifting priors towards more optimism/pessimism on exploitation rates of players. First, we look at the case in which some players' priors are shifted within our RLMPNE concept. Then we examine how RLMPNE with common priors compares with rational expectations. In particular, we show how optimism/pessimism affects the intensity of the tragedy of the commons.

\subsection{Increasing Optimism/Pessimism}

Our goal is to apply our analysis to problems in which priors at time 0 can be exogenously altered. An example of exogenously altering $\Xi$ can be found in the case of environmental 
campaigns which use novel expert information about laws of renewal of natural resources and aims at shifting the priors of players. In particular, we are interested in understanding how bias of shifted priors towards optimism vs. pessimism alters exploitation strategies. To this end, in this section we focus on prior distributions that are comparable in terms of the direction of belief bias they entail. In particular, assume that $\xi, \underline{\xi}$, and $\bar{\xi}$ are related through a strict first-order stochastic dominance (FOSD) relationship with $\underline{\xi} \prec_{F O S D} \xi \prec_{F O S D} \bar{\xi}$, while, for simplicity, assume that $\theta$ is not a parameter vector, but a single parameter. ${ }^{11}$ By the definition of strict FOSD, ${ }^{12}$

$$
\underline{\xi} \prec_{F O S D} \xi \prec_{F O S D} \bar{\xi} \Leftrightarrow \int_{\Theta} h(\theta) \underline{\xi}(\theta) \mathrm{d} \theta<\int_{\Theta} h(\theta) \xi(\theta) \mathrm{d} \theta<\int_{\Theta} h(\theta) \bar{\xi}(\theta) \mathrm{d} \theta
$$

for all strictly increasing functions $h$ on $\Theta$. Assuming that $\mu^{\prime}>0$, since

$$
\int_{\Theta} \mu(\theta) \underline{\xi}(\theta) \mathrm{d} \theta<\int_{\Theta} \mu(\theta) \xi(\theta) \mathrm{d} \theta<\int_{\Theta} \mu(\theta) \bar{\xi}(\theta) \mathrm{d} \theta
$$

if priors are initially $\xi$, then $\underline{\xi}$ represents a pessimistic shift in priors and $\bar{\xi}$ represents an optimistic shift in priors, provided that the equilibrium law of motion for the resource stock, $k$, intersects the $45^{\circ}$ line at values for $k$ above 1 , for any realization of the shock, $\eta_{t}$. To see why this technical requirement should hold in order to justify the above interpretation of optimism/pessimism related to inequality (14), recall that the law of motion without exploitation is $k_{t+1}=B k_{t}^{\eta_{t}}$, which has fixed point $K^{*}\left(\eta_{t}\right)=B^{1 /\left(1-\eta_{t}\right)}$. Higher values of $\eta_{t}$ are associated with higher $K^{*}\left(\eta_{t}\right)$, an optimistic prospect, only if $B>1$. So, without exploitation, setting $B>1$ guarantees that parameter beliefs that imply a higher value of $\int_{\Theta} \mu(\theta) \xi(\theta) \mathrm{d} \theta$ are optimistic. Yet, in equilibrium, part of the resource is consumed, so the implied fixed point of $k$ conditional on any $\eta_{t}$ is lower than $K^{*}\left(\eta_{t}\right)=B^{1 /\left(1-\eta_{t}\right)}$.

11While Proposition 1 holds for cases in which $\theta$ is a multidimensional vector, the comparisons performed in this section rely upon the concept of stochastic dominance. Avoiding the technicalities of multidimensional stochastic dominance is the main reason for assuming that $\theta$ is a single parameter in this section.

12 For the definition of strict FOSD, see, for example, Jackson and Rogers (2007, p. 6). 
Specifically, the equilibrium law of motion of $k$ is $k_{t+1}=B \cdot\left[L\left(\Xi_{t}\right) k_{t}\right]^{\eta_{t}}$, in which $L\left(\Xi_{t}\right) \equiv$ $1-\sum_{j=1}^{N} c^{j}\left(\Xi_{t}\right)$. For any given $\eta_{t} \in(0,1)$ and any $\Xi_{t}$, the implied fixed point of the law of motion $k_{t+1}=B \cdot\left[L\left(\Xi_{t}\right) k_{t}\right]^{\eta_{t}}$ is $K^{*}\left(\eta_{t} ; \Xi_{t}\right)=B \cdot\left[L\left(\Xi_{t}\right) B\right]^{1 /\left(1-\eta_{t}\right)}$. So, a loose sufficient condition for guaranteeing $K^{*}\left(\eta_{t} ; \Xi_{t}\right)>1$ for all $\eta_{t}$ is $L\left(\Xi_{t}\right) B>1$. Assumption 1 guarantees that $L\left(\Xi_{t}\right) B>1$ holds for any belief profile $\Xi_{t}$, in order to secure an a-priori optimistic shift if $\xi_{t} \prec_{F O S D} \bar{\xi}_{t}$ and $\mu^{\prime}>0$.

Assumption 1 Let $\mu\left(\theta_{\mathrm{inf}}\right)>0$, in which $\theta_{\mathrm{inf}} \equiv \inf (\Theta)$, and let $\Xi^{\mathrm{inf}}$ denote $a$ belief profile in which $\xi_{i}^{\inf }(\theta)=1$, if $\theta=\theta_{\mathrm{inf}}$, and $\xi_{i}^{\inf }(\theta)=0$, if $\theta \neq \theta_{\mathrm{inf}}$, for all $i \in\{1, \ldots, N\}$. Then,

$$
B>1+N\left[\frac{1}{\delta \mu\left(\theta_{\text {inf }}\right)}-1\right]=\frac{1}{L\left(\Xi^{\text {inf }}\right)}
$$

If (15) holds and $\mu^{\prime}>0$, then $L\left(\Xi_{t}\right) B>1$ for all $\Xi_{t}$, since, for any $\Xi, \xi_{i}^{\text {inf }} \prec_{F O S D} \xi_{i}$, for all $i \in\{1, \ldots, N\}$, which implies $\mu\left(\theta_{\text {inf }}\right)<\int_{\Theta} \mu(\theta) \xi_{i}(\theta) \mathrm{d} \theta$. In turn, equation (9) implies $L\left(\Xi^{\text {inf }}\right)<L(\Xi)$ for all $\Xi$, guaranteeing that $L(\Xi) B>1$ and $K^{*}(\eta ; \Xi)>1$ for all $\eta$ and all $\Xi$.

Proposition 2 reveals the impact of shifts in any player's priors on the strategies of other players and on the aggregate exploitation rate.

Proposition 2 Let $\mu^{\prime}>0$, and $\underline{\xi}^{i} \prec_{F O S D} \xi^{i} \prec_{F O S D} \bar{\xi}^{i}$ for some $i \in\{1, \ldots, N\}$. Then,

$$
\begin{aligned}
\text { (i) } c^{i}(\underline{\Xi}) & >c^{i}(\Xi)>c^{i}(\bar{\Xi}) \\
\text { (ii) } c^{j}(\underline{\Xi}) & <c^{j}(\Xi)<c^{j}(\bar{\Xi}) \quad \text { for all } j \in\{1, \ldots N\} \text { with } j \neq i \\
\text { (iii) } \sum_{j=1}^{N} c^{j}(\underline{\Xi}) & >\sum_{j=1}^{N} c^{j}(\Xi)>\sum_{j=1}^{N} c^{j}(\bar{\Xi})
\end{aligned}
$$


in which

$$
\Xi \equiv\left(\xi_{t}^{1}, \ldots, \xi_{t}^{i-1}, \underline{\xi}^{i}, \xi_{t}^{i+1}, \ldots, \xi_{t}^{N}\right) \quad \text { and } \quad \bar{\Xi} \equiv\left(\xi_{t}^{1}, \ldots, \xi_{t}^{i-1}, \bar{\xi}^{i}, \xi_{t}^{i+1}, \ldots, \xi_{t}^{N}\right)
$$

Proof Since $\mu^{\prime}>0, \int_{\Theta} \mu(\theta) /[1-\delta \mu(\theta)] \underline{\xi}(\theta) \mathrm{d} \theta<\int_{\Theta} \mu(\theta) /[1-\delta \mu(\theta)] \xi(\theta) \mathrm{d} \theta<$ $\int_{\Theta} \mu(\theta) /[1-\delta \mu(\theta)] \bar{\xi}(\theta) \mathrm{d} \theta$. So, inequalities (i), (ii), and (iii) are derived immediately from equation (9).

Under Assumption 1 (which holds throughout the remaining analysis), inequality (i) of Proposition 2 states that if a player $i$ becomes pessimistic $\left(\underline{\xi}^{i} \prec_{F O S D} \xi^{i}\right)$, then her exploitation rate rises. This behavior depends on perceived and envisaged growth dynamics of $k$ from each player's perspective. Below we examine whether these perceived dynamics of $k$ also depend on initial conditions.

\subsubsection{Pessimistic belief shifts, actual vs perceived dynamics, and initial conditions}

The importance of Assumption 1 is that the parametric value of parameter $B$ is high enough to guarantee that $K^{*}(\eta ; \Xi)>1$ for all $\eta$ and all $\Xi$. Proposition 2 gives illuminating insights, as we know that $k_{t}-\sum_{i=1}^{N} C^{i}\left(k_{t} ; \Xi\right)=\left[1-\sum_{i=1}^{N} c^{i}(\Xi)\right] k_{t}$. Consider the case of $N \geq 2$ and heterogeneous beliefs, with $\underline{\xi}^{i} \prec_{F O S D} \xi^{i}$ for some $i \in\{1, \ldots, N\}$. For any given $\eta_{t} \in(0,1)$, let the original equilibrium law of motion be denoted by $k_{t+1}=F^{1}\left(k_{t}\right)$, and let the equilibrium law of motion after one player's pessimistic shift be denoted by $k_{t+1}=F^{2}\left(k_{t}\right)$. In this case, inequality (iii) of Proposition 2 implies that,

$$
F^{1}\left(k_{t}\right) \equiv B \cdot\left[1-\sum_{i=1}^{N} c^{i}(\Xi)\right]^{\eta_{t}} k^{\eta_{t}}>B \cdot\left[1-\sum_{i=1}^{N} c^{i}(\Xi)\right]^{\eta_{t}} k^{\eta_{t}} \equiv F^{2}\left(k_{t}\right) .
$$

The relationship given by (16) is depicted by Figure 1 . The two fixed points of $F^{1}\left(k_{t}\right)$ and $F^{2}\left(k_{t}\right)$ are denoted by $k_{i}$, with $k_{i}=F^{i}\left(k_{i}\right), i=1,2$. Assumption 1 implies $B$. 
$\left[1-\sum_{i=1}^{N} c^{i}(\underline{\Xi})\right]>1$, which further implies $k_{2}=\left\{B \cdot\left[1-\sum_{i=1}^{N} c^{i}(\underline{\Xi})\right]^{\eta_{t}}\right\}^{1 /\left(1-\eta_{t}\right)}>1$, since $\eta_{t} \in(0,1)$. Because $k_{2}>1,(16)$ implies $k_{1}>k_{2}>1$. Figure 2 also shows that actual growth rates, $\ln \left[F^{i}\left(k_{t}\right)\right]-\ln \left(k_{t}\right), i=1,2$, are always lower if at least one player's beliefs shift to pessimism for any given $\eta_{t}$.

Players cannot rationalize their strategies by guessing the actual dynamics of $k$, because they are uninformed about $\theta^{*}$. Players rationalize their strategies based on their own anticipations about the dynamics of $k$. So, consider a player who experiences a pessimistic belief shift in the sense that $\bar{\eta} \equiv \int_{\Theta} \mu(\theta) \xi(\theta) \mathrm{d} \theta$ drops from value $\bar{\eta}_{1}$ to $\bar{\eta}_{2}<\bar{\eta}_{1}$. Denote the subjective law of motion before and after the belief shift by $H^{1}(k)$ and $H^{2}(k)$. We examine conditions under which the anticipated growth rate of the perceived law of motion will drop after the pessimistic shift, so

$$
\begin{aligned}
& E\left\{\ln \left[H^{2}\left(k_{t}\right)\right]\right\}-\ln \left(k_{t}\right)<E\left\{\ln \left[H^{1}\left(k_{t}\right)\right]\right\}-\ln \left(k_{t}\right) \Leftrightarrow \\
& \quad \Leftrightarrow \ln \left(k_{t}\right)>\frac{\bar{\eta}_{2} \ln \left[1-\sum_{i=1}^{N} c^{i}(\Xi)\right]-\bar{\eta}_{1} \ln \left[1-\sum_{i=1}^{N} c^{i}(\Xi)\right]}{\bar{\eta}_{1}-\bar{\eta}_{2}} \equiv \ln \left(k_{\text {cutoff }}\right)
\end{aligned}
$$

From (17) it is ambiguous whether $\ln \left(k_{\text {cutoff }}\right)$ is positive or negative, i.e., if $k_{\text {cutoff }}$ is greater or smaller than unity. Denote the zero-anticipated-growth points (fixed points) of $H^{1}(k)$ and $H^{2}(k)$, by $k_{p 1}$ and $k_{p 2}$ (perceived fixed points), and notice that, due to Assumption 1 , $k_{p 1}, k_{p 2}>1$. After some algebra, we can verify that,

$$
\ln \left(k_{p 1}\right)-\ln \left(k_{p 2}\right)=\frac{\bar{\eta}_{1}-\bar{\eta}_{2}}{\left(1-\bar{\eta}_{1}\right)\left(1-\bar{\eta}_{2}\right)} \ln \left\{B\left[1-\sum_{i=1}^{N} c^{i}(\Xi)\right]\right\}-\ln \left(k_{\text {cutoff }}\right) .
$$

Due to Assumption 1, the first term on the right-hand side of (18) is always strictly positive, since $\bar{\eta}_{2}<\bar{\eta}_{1}$. Equation (18) involves three cases to examine. In Case $1, k_{\text {cutoff }}<1$, so $k_{p 1}>k_{p 2}$. In Case 2 , depicted by Figures 3 and $4, k_{\text {cutoff }}>1$, but $B$ is large enough so that $k_{p 1}>k_{p 2}$. As it is obvious from Figures 3 and 4 , if $k_{0}<k_{\text {cutoff }}$, then the anticipated growth 
rate may temporalily increase, despite that the envisaged long-run fixed point is lower (the same holds in Case 1 if $\left.k_{0}<k_{\text {cutoff }}\right)$. Case 3 , implied by (18), is having $k_{\text {cutoff }}>1$ being large enough so that $1<k_{p 1}<k_{p 2}<k_{\text {cutoff }}$ In Case 3 , which is rather extreme, relying on values of aggregate exploitation rates very close to unity, a decrease in $\bar{\eta}$ implies more favorable anticipated temporary and longer-term dynamics.

In a nutshell, different initial conditions, or extreme exploitation rates, may affect anticipated temporary and longer-term growth rates of $k$. Differences in the anticipated growth rates can make the interpretation of pessimism (a reduction in $\int_{\Theta} \mu(\theta) \xi_{i}(\theta) \mathrm{d} \theta$ ) ambiguous, depending on initial conditions. Nevertheless, reasonably large values of $B$, guaranteeing that near-steady state and transitional values of $k$ are well above unity, and initial conditions above unity, preserve the subjective interpretation of optimism/pessimism in this model, justifying our focus on interpreting first-order stochastic dominance such as $\underline{\xi}^{i} \prec_{F O S D} \xi^{i}$, as a pessimistic belief shift. Importantly, as depicted by Figures 1 and 2, Assumption 1 guarantees that $\underline{\xi}^{i} \prec_{F O S D} \xi^{i}$ always implies unfavorable actual growth rates for $k$.

Proposition 2 says that regardless of whether anticipated temporary and longer-term dynamics are favorable or not, the player experiencing the direct pessimistic shift will always raise her exploitation rate. In the case of $N \geq 2$ and heterogeneous beliefs, with $\underline{\xi}^{i} \prec_{F O S D}$ $\xi^{i}$ for some $i \in\{1, \ldots, N\}$, inequality (i) of Proposition 2 distinguishes the behavior of the player $i$, whose priors are shifted alone towards pessimism. Specifically, player $i$ alone consumes more, despite the strategic responses of all other players $j \neq i$. Interestingly, the strategic response of all other players, $j \neq i$, to the pessimistic shift of $i$ 's priors implies lower exploitation rates for each $j \in\{1, \ldots, N\}$ with $j \neq i$ (see inequality (ii) of Proposition 2). So, under RLMPNE, each player $j \neq i$ internalizes that $i$ 's new strategy will be directly reducing the accumulated stock of the resource in any future period. 
As future consumption of the resource is a normal good for all players, each player $j \neq i$ reduces her own extraction rate and contributes to the conservation of the resource. Yet, inequality (iii) of Proposition 2 shows that, on aggregate, the conservation incentive which is implied inequality (ii) of Proposition 2 is not strong enough. The overall outcome of shifting at least one player's priors towards pessimism is a collective anti-conservation incentive.

Despite the impact of pessimism on aggregate exploitation, inequality (ii) of Proposition 2 reveals that the noncooperative RLMPNE solution contains some strategic conservation incentives. These strategic conservation incentives give a chance for players to mitigate the commons problem. The section below studies this possibility under identical priors.

\subsection{Identical Priors, Belief Bias, and the Intensity of the Com- mons Problem}

The commons problem is the aggregate-exploitation rate's tendency to increase as more players are added to the noncooperative setup. In the process of studying this tendency under heterogeneous beliefs, one must characterize the prior-beliefs identity of any player added to the game. ${ }^{13}$ The assumption of identical priors overcomes this identity-specification problem of additional players. Identical priors and jointly identical shifts in these priors allow us to examine how increasing pessimism across all players affects the intensity of the commons problem. Specifically, if the aggregate noncooperative exploitation rate is denoted by $G$, the intensity of the commons problem is captured by the positively-valued derivative $\partial G / \partial N$, in which $N$ is the number of identical players. We wish to examine whether a pessimistic shift in beliefs increases or decreases the value of $\partial G / \partial N$. An increase in the

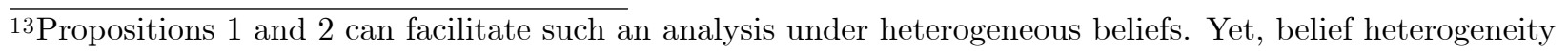
makes it difficult to obtain intuitive generalizations about how beliefs affect the intensity of the commons problem. Under heterogeneous beliefs, one would need to compare equilibrium extraction rates with a social-planner's solution under heterogeneous beliefs. This cumbersome task is beyond the scope of this paper. 
value of $\partial G / \partial N$ due to a pessimistic belief shift reflects an exacerbation of the commons problem (see also Antoniadou et al. 2013, Proposition 3, p. 36).

Let an identical-priors belief profile be denoted by $\Xi^{I P}$, with $\xi_{i}=\xi$ for all $\xi_{i} \in \Xi^{I P}$, and for all $i \in\{1, \ldots, N\}$. Given $\Xi^{I P}$, after some algebra, equation (9) implies that the aggregate exploitation rate is given by,

$$
\sum_{j=1}^{N} c^{j}\left(\Xi^{I P}\right) \equiv G\left(N, c^{o}(\xi)\right)=\frac{N c^{o}(\xi)}{1+(N-1) c^{o}(\xi)},
$$

in which the functional $c^{o}(\xi)$ is the "cooperative" exploitation rate given by,

$$
c(\Xi)=c^{o}(\xi)=\left(\int_{\Theta} \frac{1}{1-\delta \mu(\theta)} \xi(\theta) d \theta\right)^{-1} .
$$

Equation (20) can be calculated by setting $N=1$ in equation (9), since the cooperative coalition will control the resource as if a single player, distributing $c^{o}(\xi) / N$ to each player for consumption. The term $c^{o}(\xi)$ in equation (19) distinguishes the impact of the model's fundamentals on the cooperative solution. Yet, the interaction of $c^{o}(\xi)$ with the number of players, $N$, sheds light on the strength of strategic incentives that arise in the noncooperative case. In particular, this interaction helps in identifying conditions under which jointly identical shifts in identical priors towards pessimism exacerbate the commons problem in the noncooperative game. ${ }^{14}$

As equation (20) implies, increasing pessimism for all players makes the cooperative exploitation rate, $c^{o}(\xi)$, to rise, as long as $\mu^{\prime}>0 .{ }^{15}$ We focus on the magnitude of increase in the cooperative rate $c^{o}(\xi)$ caused by a shift towards pessimism. This focus on the magnitude of change in $c^{o}(\xi)$ is informative, since the way beliefs affect the cooperative exploitation

14The commons problem is straightforward to verify from equation (19). Increasing $N$, i.e. the number of players with the same priors, always leads to higher aggregate exploitation rate, $G\left(N, c^{o}(\xi)\right)$.

15 Recall from our analysis above that such a shift in priors towards pessimism is captured by $\underline{\xi} \prec_{F O S D} \xi$, in which $\xi$ is the initial priors and $\underline{\xi}$ is beliefs after the shift. The result is $c^{o}(\underline{\xi})>c^{o}(\xi)$. 
rate is distinct from the way strategic interaction affects the aggregate noncooperative exploitation rate $G\left(N, c^{o}(\xi)\right)$. In particular, equation (19) implies,

$$
\frac{\partial^{2} G\left(N, c^{o}(\xi)\right)}{\partial N \partial c^{o}(\xi)}=\left[1+(N-1) c^{o}(\xi)\right]^{-3} \cdot\left[1-c^{o}(\xi)-N c^{o}(\xi)\right] .
$$

Based on (21), Proposition 3 summarizes the conditions under which a shift in common priors exacerbates the commons problem.

Proposition 3 Let $\mu^{\prime}>0$, and let all players have identical priors, $\Xi^{I P}$. If

$$
\underbrace{\frac{1-c^{o}(\xi)}{N}}_{\text {cooperative plowback rate per player }}>\underbrace{c^{o}(\xi)}_{\text {cooperative exploitation rate }} \Leftrightarrow \frac{1-c^{o}(\xi)}{c^{o}(\xi)}>N,
$$

then an identical shift in all players' priors towards pessimism exacerbates the commons problem.

\section{Proof Immediate from (21).}

Proposition 3 says that the initial number of players is crucial for determining whether an increase in pessimism exacerbates the commons problem. A "small" number of players may trigger such an exacerbating effect of increases in pessimism as long as initial priors imply a "small" cooperative exploitation rate, too. What is meant by "small" for the initial number of players, $N$, and the initial cooperative exploitation rate, $c^{o}(\xi)$, is determined by equation (22): once the initial cooperative plowback rate divided by the initial number of players is larger than the initial cooperative exploitation rate, an increase in pessimism exacerbates the commons problem.

The intuition behind the crucial role played by the number of players in Proposition 3 is revealed by Proposition 2. In Proposition 2 we have seen that once pessimism increases for one player, each other player decreases her own exploitation rate in order to conserve the 
resource. For players with identical priors, if pessimism increases for each and every player by the same degree, then equation (19) reveals that each player's individual exploitation rate will increase, too. ${ }^{16}$ Yet, due to the strategic conservation response revealed by Proposition 2, as the number of players increases, the increase in the individual exploitation rate due to increases in $c^{o}(\xi)$ falls. ${ }^{17}$ Proposition 3 shows that, after a critical number of players indicated through equation (22), the strategic-conservation-response effect dominates, and the commons problem is mitigated.

The exact mechanics described by Propositions 1 through 3 are specific to the particular workable example. Nevertheless, the qualitative direction of strategic incentives pointed out by these mechanics is both intuitive and likely to characterize more general settings. The most important implication of Propositions 1 through 3 is the tradeoff between conservation incentives and the type of strategic individualism that leads to the commons problem.

\section{Conclusion}

We have built a workable game in which players rationally learn about renewal prospects of a commonly exploited natural resource. Despite the high complexity of games in which a state variable is accumulated in a stochastic environment under rational learning and belief heterogeneity, we have managed to construct an example which delivers explicit solutions for general prior distributions.

We have examined how shifts in players' priors towards pessimism affect strategic exploitation. We have also focused on the effects of such shifts on the commons problem.

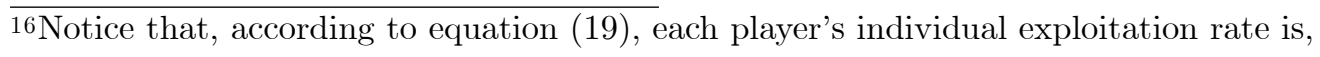

$$
\frac{G\left(N, c^{o}(\xi)\right)}{N}=\frac{c^{o}(\xi)}{1+(N-1) c^{o}(\xi)},
$$

which is increasing in $c^{o}(\xi)$.

17This is verifiable from equation (19), which implies that $\partial^{2}\left[G\left(N, c^{o}(\xi)\right) / N\right] /\left[\partial N \partial c^{o}(\xi)\right]<0$. 
We have found that if the initial number of players is high, then strategic conservation incentives may dominate, with increases in pessimism mitigating but not eliminating the commons problem.

Our results may give a pointer to regulation policies regarding noncooperative exploitation of common resources. If access to common-property exploitation is granted to additional players in a period that pessimism about the natural renewal prospects of a resource rises, then the commons problem may arise stronger, if the original number of players and the original exploitation rate are both small. Such situations may arise in periods that environmental campaigns cause pessimistic shifts to such priors of investors, companies with exploitation rights, countries, even consumers, by presenting novel evidence irrelevant to the everyday process of learning by observation.

Regarding our framework of analysis, our study has assumed that each player knows the prior beliefs of all other players. If, for example, players are countries, then the media may have the beliefs of each country fully revealed, validating our assumption. Nevertheless, our study has left games in which players are uninformed about the beliefs of other players unexplored. Certainly, the assumption of not knowing other players' beliefs is more suitable for some applications. The study of second-order learning (with each player updating her beliefs about the beliefs of other players) is an extension for future research that our framework may accommodate. 


\section{Appendix - Proofs}

The proof of Proposition 1 relies on Lemmata 1 through 3, which are separate results. We state and prove Lemmata 1 and 3 below, while the proof of Lemma 2 appears in the literature. So, we state Lemma 2 and provide an appropriate citation for its proof.

Lemma 1 For any function $h: \mathbb{R} \rightarrow \mathbb{R}$ and any given prior distribution $\xi_{0}$, for all $t \in\{1,2, \ldots\}$ and $\left\{\xi_{s}\right\}_{s=0}^{t-1}$ generated through

$$
\xi_{\tau+1}(\theta \mid \eta)=\frac{\phi(\eta \mid \theta) \xi_{\tau}(\theta)}{\int_{\Theta} \phi(\eta \mid x) \xi_{\tau}(x) \mathrm{d} x}, \quad \tau=0,1, \ldots,
$$

from any sequence $\left\{\eta_{s}\right\}_{s=0}^{t-1}$ of independent draws of the shock $\eta$, the conditional expectation

$$
\begin{aligned}
& E_{0}\left(\prod_{s=0}^{t-1} h\left(\eta_{s}\right) \mid \xi_{0}, \text { update governed by }(23)\right) \equiv \\
& \equiv \int_{\mathcal{H}} \int_{\Theta} \cdots \int_{\mathcal{H}} \int_{\Theta} \int_{\mathcal{H}} \int_{\Theta} \prod_{s=0}^{t-1} h\left(\eta_{s}\right) \phi\left(\eta_{t-1} \mid \theta_{t-1}\right) \xi_{t-1}\left(\theta_{t-1}\right) \mathrm{d} \theta_{t-1} \mathrm{~d} \eta_{t-1} \times \\
& \quad \times \phi\left(\eta_{t-2} \mid \theta_{t-2}\right) \xi_{t-2}\left(\theta_{t-2}\right) \mathrm{d} \theta_{t-2} \mathrm{~d} \eta_{t-2} \times \cdots \times \phi\left(\eta_{0} \mid \theta_{0}\right) \xi_{0}\left(\theta_{0}\right) \mathrm{d} \theta_{0} \mathrm{~d} \eta_{0}= \\
& =\int_{\Theta}\left[\int_{\mathcal{H}} h(\eta) \phi(\eta \mid \theta) \mathrm{d} \eta\right]^{t} \xi_{0}(\theta) \mathrm{d} \theta
\end{aligned}
$$

assumming that appropriate integrability conditions hold in (24) for all $t \in$ $\{1,2, \ldots\}$.

\section{Proof of Lemma 1}

We express $\xi_{t-1}$ as a function of $\xi_{t-2}$, according to the Bayesian update of beliefs given by (23), and we substitute it into the LHS of (24),

$$
\int_{\mathcal{H}} \int_{\Theta} \cdots \int_{\mathcal{H}} \int_{\Theta} \int_{\mathcal{H}} \int_{\Theta} \prod_{s=0}^{t-1} h\left(\eta_{s}\right) \phi\left(\eta_{t-1} \mid \theta_{t-1}\right) \frac{\phi\left(\eta_{t-2} \mid \theta_{t-1}\right) \xi_{t-2}\left(\theta_{t-1}\right)}{\int_{\Theta} \phi\left(\eta_{t-2} \mid x\right) \xi_{t-2}(x) \mathrm{d} x} \times
$$




$$
\begin{aligned}
\times & \mathrm{d} \theta_{t-1} \mathrm{~d} \eta_{t-1} \phi\left(\eta_{t-2} \mid \theta_{t-2}\right) \xi_{t-2}\left(\theta_{t-2}\right) \mathrm{d} \theta_{t-2} \mathrm{~d} \eta_{t-2} \times \cdots \times \phi\left(\eta_{0} \mid \theta_{0}\right) \xi_{0}\left(\theta_{0}\right) \mathrm{d} \theta_{0} \mathrm{~d} \eta_{0}= \\
=\int_{\mathcal{H}} \int_{\Theta} & \cdots \int_{\mathcal{H}} \int_{\mathcal{H}} \int_{\Theta} \prod_{s=0}^{t-1} h\left(\eta_{s}\right) \phi\left(\eta_{t-1} \mid \theta_{t-1}\right) \phi\left(\eta_{t-2} \mid \theta_{t-1}\right) \xi_{t-2}\left(\theta_{t-1}\right) \mathrm{d} \theta_{t-1} \times \\
& \times \mathrm{d} \eta_{t-1} \mathrm{~d} \eta_{t-2} \phi\left(\eta_{t-3} \mid \theta_{t-3}\right) \xi_{t-3}\left(\theta_{t-3}\right) \mathrm{d} \theta_{t-3} \mathrm{~d} \eta_{t-3} \times \cdots \times \phi\left(\eta_{0} \mid \theta_{0}\right) \xi_{0}\left(\theta_{0}\right) \mathrm{d} \theta_{0} \mathrm{~d} \eta_{0},
\end{aligned}
$$

i.e., $\xi_{t-1}$ has been cancelled from the expression. Continuing in this way up to period 0 , the LHS of (24) becomes

$$
\int_{\mathcal{H}} \cdots \int_{\mathcal{H}} \int_{\mathcal{H}} \int_{\Theta} \prod_{s=0}^{t-1} h\left(\eta_{s}\right) \phi\left(\eta_{s} \mid \theta_{t-1}\right) \xi_{0}\left(\theta_{t-1}\right) \mathrm{d} \theta_{t-1} \mathrm{~d} \eta_{t-1} \mathrm{~d} \eta_{t-2} \times \cdots \times \mathrm{d} \eta_{0}
$$

and because $\eta$ 's are independent over time, this last expression is equals the result given by $(24)$.

Lemma 2 (matrix determinant lemma) Let $A$ be an $N \times N$ nonsingular matrix, and $x, y$ be any $N \times 1$ vectors. Then,

$$
\operatorname{det}\left(A+x \cdot y^{T}\right)=\left(1+y^{T} \cdot A^{-1} \cdot x\right) \cdot \operatorname{det}(A) .
$$

\section{Proof of Lemma 2}

See Harville (1997, p. 416, Theorem 18.1.1 and Corollaries 18.1.2 and 18.1.3). 
Lemma 3 Let the $N \times N$ linear system

$$
\mathbf{A} \cdot\left[\begin{array}{c}
x_{1} \\
x_{2} \\
\vdots \\
x_{N}
\end{array}\right]=\left[\begin{array}{c}
1 \\
1 \\
\vdots \\
1
\end{array}\right]
$$

in which,

$$
\mathbf{A} \equiv\left[\begin{array}{cccc}
a_{1} & 1 & \cdots & 1 \\
1 & a_{2} & \cdots & 1 \\
\vdots & \vdots & \ddots & \vdots \\
1 & 1 & \cdots & a_{N}
\end{array}\right]
$$

with $a_{i} \neq 1$ for all $i \in\{1, \ldots, N\}$. Then, the solution to this system (denoted by a star) is unique with,

$$
x_{i}^{*}=\frac{\frac{1}{a_{i}-1}}{1+\sum_{j=1}^{N} \frac{1}{a_{j}-1}}, \quad \text { for all } i \in\{1, \ldots, N\} .
$$

\section{Proof of Lemma 3}

Simple substitution of (26) into (25) proves the validity of the solution. The condition $a_{i} \neq 1$ for all $i \in\{1, \ldots, N\}$ guarantees linear independence among all rows (and columns) of $A$, which implies the nonsingularity of $\mathbf{A}$, which, in turn, proves the uniqueness of (26), proving the Lemma. Yet, below we provide the steps that lead to identifying this solution, which may prove useful for characterizing similar results in other resource-extraction games with similar structure. ${ }^{18}$

Pick any $i \in\{1, . ., N\}$, and notice that (25) implies,

$$
x_{i}^{*}=\mathbf{0}_{\mathbf{1}_{i}}^{T} \cdot \mathbf{A}^{-1} \cdot \mathbf{1}_{N},
$$

18This proof first appeared in Koulovatianos (2010, pp. 27-29), which is an early version of this paper. 
in which $\mathbf{1}_{N}$ is an $N \times 1$ vector of ones, and $\mathbf{0}_{\mathbf{1}_{i}}$ is an $N \times 1$ vector of zeros, with the sole exception that its $i$-th element is equal to 1 . The matrix determinant lemma (Lemma 2) implies that

$$
\operatorname{det}\left(\mathbf{A}-\mathbf{1}_{N} \cdot \mathbf{0}_{\mathbf{1}_{i}}^{T}\right)=\left(1-\mathbf{0}_{\mathbf{1}_{i}}^{T} \cdot \mathbf{A}^{-1} \cdot \mathbf{1}_{N}\right) \cdot \operatorname{det}(\mathbf{A}) .
$$

Setting,

$$
\mathbf{A}_{i} \equiv \mathbf{A}-\mathbf{1}_{N} \cdot \mathbf{0}_{\mathbf{1}_{i}}^{T}
$$

and combining (27) with (28), we obtain

$$
x_{i}^{*}=1-\frac{\operatorname{det}\left(\mathbf{A}_{i}\right)}{\operatorname{det}(\mathbf{A})} .
$$

Equation (29) implies that, in order to characterize $x_{i}^{*}$, we must first characterize $\operatorname{det}\left(\mathbf{A}_{i}\right)$ and $\operatorname{det}(\mathbf{A})$. We start from characterizing $\operatorname{det}(\mathbf{A})$. Let

$$
\tilde{\mathbf{A}} \equiv \mathbf{A}-\mathbf{1}_{N} \cdot \mathbf{1}_{N}^{T}
$$

which implies that $\tilde{\mathbf{A}}$ is a diagonal matrix. Denoting the $i$-th diagonal element of $\tilde{\mathbf{A}}$ by $\operatorname{diag}(\tilde{\mathbf{A}})_{i}$, it is,

$$
\operatorname{diag}(\tilde{\mathbf{A}})_{i}=a_{i}-1
$$

Applying again the matrix determinant lemma (Lemma 2),

$$
\operatorname{det}(\mathbf{A})=\operatorname{det}\left(\tilde{\mathbf{A}}+\mathbf{1}_{N} \cdot \mathbf{1}_{N}^{T}\right)=\left(1+\mathbf{1}_{N}^{T} \cdot \tilde{\mathbf{A}}^{-1} \cdot \mathbf{1}_{N}\right) \cdot \operatorname{det}(\tilde{\mathbf{A}}),
$$

which implies

$$
\operatorname{det}(\mathbf{A})=\left(1+\sum_{i=1}^{N} \frac{1}{\operatorname{diag}(\tilde{\mathbf{A}})_{i}}\right) \cdot \prod_{i=1}^{N} \operatorname{diag}(\tilde{\mathbf{A}})_{i} .
$$

In order to characterize $\operatorname{det}\left(\mathbf{A}_{i}\right)$, we use the definitions of $\mathbf{A}_{i}$ and $\tilde{\mathbf{A}}$, noticing that $\mathbf{A}_{i}-\tilde{\mathbf{A}}=\mathbf{1}_{N} \cdot\left(\mathbf{1}_{N}^{T}-\mathbf{0}_{\mathbf{1}_{i}}^{T}\right)$, which implies,

$$
\mathbf{A}_{i}=\tilde{\mathbf{A}}+\mathbf{1}_{N} \cdot \mathbf{1}_{\mathbf{0}_{i}}^{T},
$$


in which $\mathbf{1}_{\mathbf{0}_{i}}$ is an $N \times 1$ vector of ones, with the sole exception that its $i$-th element is equal to 0. Combining (32) with the matrix determinant lemma (Lemma 2),

$$
\operatorname{det}\left(\mathbf{A}_{i}\right)=\operatorname{det}\left(\tilde{\mathbf{A}}+\mathbf{1}_{N} \cdot \mathbf{1}_{\mathbf{0}_{i}}^{T}\right)=\left(1+\mathbf{1}_{\mathbf{0}_{i}}^{T} \cdot \tilde{\mathbf{A}}^{-1} \cdot \mathbf{1}_{N}\right) \cdot \operatorname{det}(\tilde{\mathbf{A}})
$$

which gives,

$$
\operatorname{det}\left(\mathbf{A}_{i}\right)=\left(1+\sum_{\substack{j=1 \\ j \neq i}}^{N} \frac{1}{\operatorname{diag}(\tilde{\mathbf{A}})_{j}}\right) \cdot \prod_{i=1}^{N} \operatorname{diag}(\tilde{\mathbf{A}})_{i} .
$$

Equation (26) is derived after combining (29) with (33), (31), and (30). The formula given by equation (26) holds for all $i \in\{1, \ldots, N\}$ since the choice of $i$ was arbitrary.

\section{Proof of Proposition 1}

Our solution approach follows Levhari and Mirman (1980). We start from deriving RLMPNE in the finite-horizon setting. Then we use the finite-horizon RLMPNE results in order to generalize them to the infinite-horizon case. The approach of Levhari and Mirman (1980) for proving the result helps in exhibiting the informational structure of the problem.

The static problem (0-period-horizon problem)

The Nash-equilibrium solution is not unique in this case, but without loss of generality we can set,

$$
c_{0}^{i}=\kappa_{i}^{(0)} k
$$

in which $\kappa_{i}^{(0)}$ is some constant with $\kappa_{i}^{(0)} \in[0,1]$ for all $i \in\{1, \ldots, N\}$, with $\Sigma_{i=1}^{N} \kappa_{i}^{(0)}=1$. (We are solving this problem recursively, so we denote the $n$-th iteration by a superscript " $(n)$ " wherever this is applicable.) In order to keep each player's problem well-defined in next iteration, so as to comply with the requirement that value functions are well-defined (notice 
that logarithmic utility implies that zero consumption in one period implies a value function equal to minus-infinity, and zero-consumption choices prevail in a Markov-perfect solution) we focus on a solution with $\kappa_{i}^{(0)} \in(0,1)$ for all $i \in\{1, \ldots, N\}$, and with $\Sigma_{i=1}^{N} \kappa_{i}^{(0)}=1$. So, the value function of the agent in the static problem is,

$$
V^{i,(0)}\left(k ; \Xi_{0}\right)=\ln (k)+\ln \left(\kappa_{i}^{(0)}\right)
$$

which is the only case with the value function not depending on $\Xi_{0}$.

\section{The 1-period-horizon problem}

The decision of player $i$ is determined by the Bellman equation,

$$
\begin{aligned}
& V^{i,(1)}\left(k ; \Xi_{0}\right)=\max _{c_{i} \geq 0}\left\{\ln \left(c_{i}\right)+\right. \\
& \left.\quad+\delta \int_{\mathcal{H}} V^{i,(0)}\left(B \cdot\left[k-c_{i}-\sum_{j \neq i} C^{j,(1)}\left(k ; \Xi_{0}\right)\right]^{\eta} ; \Xi_{1}(\cdot \mid \eta)\right)\left[\int_{\Theta} \phi(\eta \mid \theta) \xi_{0}^{i}(\theta) \mathrm{d} \theta\right] \mathrm{d} \eta\right\}
\end{aligned}
$$

so, using (35),

$$
\begin{aligned}
& V^{i,(1)}\left(k ; \Xi_{0}\right)=\max _{c_{i} \geq 0}\left\{\ln \left(c_{i}\right)+\right. \\
& \left.\quad+\delta \int_{\mathcal{H}} \eta \ln \left[k-c_{i}-\sum_{j \neq i} C^{j,(1)}\left(k ; \Xi_{0}\right)\right]\left[\int_{\Theta} \phi(\eta \mid \theta) \xi_{0}^{i}(\theta) \mathrm{d} \theta\right] \mathrm{d} \eta+\delta\left[\ln (B)+\ln \left(\kappa_{i}^{(0)}\right)\right]\right\}
\end{aligned}
$$

with first-order condition,

$$
\frac{1}{c_{i}}=\frac{\delta \int_{\mathcal{H}} \eta\left[\int_{\Theta} \phi(\eta \mid \theta) \xi_{0}^{i}(\theta) \mathrm{d} \theta\right] \mathrm{d} \eta}{k-c_{i}-\sum_{j \neq i} C^{j,(1)}\left(k ; \Xi_{0}\right)},
$$

which implies that, for all $i \in\{1, \ldots, N\}, C^{i,(1)}\left(k ; \Xi_{0}\right)$ is of the multiplicatively-separable form,

$$
C^{i,(1)}\left(k ; \Xi_{0}\right)=c^{i,(1)}\left(\Xi_{0}\right) \cdot k
$$


in which $\left\{c^{i,(1)}\left(\Xi_{0}\right)\right\}_{i=1}^{N}$ is the unique solution to the linear system,

$$
\left[\begin{array}{cccc}
1+\delta E_{0}\left(\eta \mid \xi_{0}^{1}\right) & 1 & \cdots & 1 \\
1 & 1+\delta E_{0}\left(\eta \mid \xi_{0}^{2}\right) & \cdots & 1 \\
\vdots & \vdots & \ddots & \vdots \\
1 & 1 & \cdots & 1+\delta E_{0}\left(\eta \mid \xi_{0}^{N}\right)
\end{array}\right]\left[\begin{array}{c}
c^{1,(1)}\left(\Xi_{0}\right) \\
c^{2,(1)}\left(\Xi_{0}\right) \\
\vdots \\
c^{N,(1)}\left(\Xi_{0}\right)
\end{array}\right]=\left[\begin{array}{c}
1 \\
1 \\
\vdots \\
1
\end{array}\right]
$$

with

$$
E_{0}\left(\eta \mid \xi_{0}^{i}\right) \equiv \int_{\mathcal{H}} \eta\left[\int_{\Theta} \phi(\eta \mid \theta) \xi_{0}^{i}(\theta) \mathrm{d} \theta\right] \mathrm{d} \eta, \quad i=1, \ldots, N
$$

Lemma 3 applies to the linear system given by (38), and it implies that (38) has a unique solution with $c^{i,(1)}\left(\Xi_{0}\right) \in(0,1)$, and $\Sigma_{i} c^{i,(1)}\left(\Xi_{0}\right) \in(0,1)$. Substituting $\left\{C^{i,(1)}\left(\Xi_{0}\right)\right\}_{i=1}^{N}$ of the multiplicatively-separable form given by (37) into (36), the Bellman equation, leads to a value function of the form,

$$
V^{i,(1)}\left(k ; \Xi_{0}\right)=\left\{1+\delta \int_{\mathcal{H}} \eta\left[\int_{\Theta} \phi(\eta \mid \theta) \xi_{0}^{i}(\theta) \mathrm{d} \theta\right] \mathrm{d} \eta\right\} \ln (k)+\kappa^{i,(1)}\left(\Xi_{0}\right),
$$

for all $i \in\{1, \ldots, N\}$, in which $\kappa^{i,(1)}\left(\Xi_{0}\right)$ is a constant that does not affect optimization in future steps. Unlike $V^{i,(0)}\left(k ; \Xi_{0}\right)$, the value function $V^{i,(1)}\left(k ; \Xi_{0}\right)$ depends on $\Xi_{0}$. Yet, we have an explicit form regarding the way $V^{i,(1)}\left(k ; \Xi_{0}\right)$ depends on $\Xi_{0}$. Most interestingly, in equation (40) the coefficient of $\ln (k)$ depends on $\xi_{0}^{i}$ only, and not on the beliefs of other individuals.

The 2-period-horizon problem

The decision of player $i$ is now determined by the Bellman equation,

$V^{i,(2)}\left(k ; \Xi_{0}\right)=\max _{c_{i} \geq 0}\left\{\ln \left(c_{i}\right)+\right.$ 


$$
\left.+\delta \int_{\mathcal{H}} V^{i,(1)}\left(B \cdot\left[k-c_{i}-\sum_{j \neq i} C^{j,(2)}\left(k ; \Xi_{0}\right)\right]^{\eta} ; \Xi_{1}(\cdot \mid \eta)\right)\left[\int_{\Theta} \phi(\eta \mid \theta) \xi_{0}^{i}(\theta) \mathrm{d} \theta\right] \mathrm{d} \eta\right\}
$$

so, using (40),

$$
\begin{aligned}
& V^{i,(2)}\left(k ; \Xi_{0}\right)=\max _{c_{i} \geq 0}\left\{\ln \left(c_{i}\right)+\right. \\
& +\delta \int_{\mathcal{H}} \eta_{0}\left\{1+\delta \int_{\mathcal{H}} \eta_{1}\left[\int_{\Theta} \phi\left(\eta_{1} \mid \theta_{1}\right) \xi_{1}^{i}\left(\theta_{1} \mid \eta_{0}\right) \mathrm{d} \theta_{1}\right] \mathrm{d} \eta_{1}\right\} \ln \left[k-c_{i}-\sum_{j \neq i} C^{j,(2)}\left(k ; \Xi_{0}\right)\right] \times \\
& \left.\quad \times\left[\int_{\Theta} \phi\left(\eta_{0} \mid \theta\right) \xi_{0}^{i}(\theta) \mathrm{d} \theta\right] \mathrm{d} \eta_{0}+\delta\left\{\ln (B)+\int_{\mathcal{H}} \kappa^{i,(1)}\left(\Xi_{1}(\cdot \mid \eta)\right)\left[\int_{\Theta} \phi(\eta \mid \theta) \xi_{0}^{i}(\theta) \mathrm{d} \theta\right] \mathrm{d} \eta\right\}\right\}
\end{aligned}
$$

subject to,

$$
\xi_{1}^{i}(\theta \mid \eta)=\frac{\phi(\eta \mid \theta) \xi_{0}^{i}(\theta)}{\int_{\Theta} \phi(\eta \mid x) \xi_{0}^{i}(x) \mathrm{d} x}, \quad i=1, \ldots, N .
$$

What is crucial to observe here is the notation about the timing of shocks. In the problem expressed by (41), each player is deciding upon a strategy in period 0, expecting both a shock $\eta_{0}$ in period 0 , after the decision has been made, and a shock $\eta_{1}$ in period 1 . Yet, it is the shock $\eta_{0}$ which will determine how the prior distribution $\xi_{0}^{i}$ will evolve to $\xi_{1}^{i}$, which is an element that the analytic form of (41) allows us to see explicitly. So, with the time horizon being expanded, we can see how prior beliefs determine what type of information is expected to arrive and also how this information is expected to be exploited.

To simplify notation, we can re-write (41) as,

$$
\begin{aligned}
V^{i,(2)}\left(k ; \Xi_{0}\right) & =\max _{c_{i} \geq 0}\left\{\ln \left(c_{i}\right)+\delta\left[E_{0}\left(\eta_{0} \mid \xi_{0}^{i}\right)+\delta E_{0}\left(\eta_{1} \eta_{0} \mid \xi_{0}^{i}\right)\right] \times\right. \\
& \left.\times \ln \left[k-c_{i}-\sum_{j \neq i} C^{j,(2)}\left(k ; \Xi_{0}\right)\right]+\delta\left[\ln (B)+E_{0}\left[\kappa^{i,(1)}(\Xi(\cdot \mid \eta))\right]\right]\right\},
\end{aligned}
$$

with

$$
E_{0}\left(\eta_{0} \mid \xi_{0}^{i}\right) \equiv \int_{\mathcal{H}} \eta_{0}\left[\int_{\Theta} \phi\left(\eta_{0} \mid \theta\right) \xi_{0}^{i}(\theta) \mathrm{d} \theta\right] \mathrm{d} \eta_{0}
$$


as in equation (39) above, and,

$$
E_{0}\left(\eta_{1} \eta_{0} \mid \xi_{0}^{i}\right) \equiv \int_{\mathcal{H}} \eta_{0} \int_{\mathcal{H}} \eta_{1}\left[\int_{\Theta} \phi\left(\eta_{1} \mid \theta_{1}\right) \xi_{1}^{i}\left(\theta_{1} \mid \eta_{0}\right) \mathrm{d} \theta_{1}\right] \mathrm{d} \eta_{1}\left[\int_{\Theta} \phi\left(\eta_{0} \mid \theta\right) \xi_{0}^{i}(\theta) \mathrm{d} \theta\right] \mathrm{d} \eta_{0}
$$

in which $\xi_{1}^{i}\left(\theta_{1} \mid \eta_{0}\right)$ is given from (42). The first-order conditions of (43) are given by,

$$
\frac{1}{c_{i}}=\delta\left[E_{0}\left(\eta_{0} \mid \xi_{0}^{i}\right)+\delta E_{0}\left(\eta_{1} \eta_{0} \mid \xi_{0}^{i}\right)\right] \frac{1}{k-c_{i}-\sum_{j \neq i} C^{j,(2)}\left(k ; \Xi_{0}\right)}
$$

which implies that, $C^{i,(1)}\left(k ; \Xi_{0}\right)$ is of the multiplicatively-separable form,

$$
C^{i,(2)}\left(k ; \Xi_{0}\right)=c^{i,(2)}\left(\Xi_{0}\right) \cdot k
$$

for all $i \in\{1, \ldots, N\}$, where $\left\{c^{i,(2)}\left(\Xi_{0}\right)\right\}_{i=1}^{N}$ is the unique solution to the linear system,

$$
\left[\begin{array}{cccc}
A^{(2)}\left(\xi_{0}^{1}\right) & 1 & \cdots & 1 \\
1 & A^{(2)}\left(\xi_{0}^{2}\right) & \cdots & 1 \\
\vdots & \vdots & \ddots & \vdots \\
1 & 1 & \cdots & A^{(2)}\left(\xi_{0}^{N}\right)
\end{array}\right] \cdot\left[\begin{array}{c}
c^{1,(2)}\left(\Xi_{0}\right) \\
c^{2,(2)}\left(\Xi_{0}\right) \\
\vdots \\
c^{N,(2)}\left(\Xi_{0}\right)
\end{array}\right]=\left[\begin{array}{c}
1 \\
1 \\
\vdots \\
1
\end{array}\right]
$$

in which

$$
A^{(2)}\left(\xi_{0}^{i}\right) \equiv 1+\delta\left[E_{0}\left(\eta_{0} \mid \xi_{0}^{i}\right)+\delta E_{0}\left(\eta_{1} \eta_{0} \mid \xi_{0}^{i}\right)\right], i \in\{1, \ldots, N\}
$$

Again, Lemma 3 guarantees that $(45)$ has a unique solution with $c^{i,(2)}\left(\Xi_{0}\right) \in(0,1)$, and $\Sigma_{i} c^{i,(2)}\left(\Xi_{0}\right) \in(0,1)$, while substitution of $\left\{C^{i,(2)}\left(\Xi_{0}\right)\right\}_{i=1}^{N}$ as given by (44) into the Bellman equation given by (43) gives a value function of the form,

$$
V^{i,(2)}\left(k ; \Xi_{0}\right)=A^{(2)}\left(\xi_{0}^{i}\right) \ln (k)+\kappa^{i,(2)}\left(\Xi_{0}\right)
$$

where $\kappa^{i,(2)}\left(\Xi_{0}\right)$ is a constant that does not affect optimization in any future step. At this point we have seen enough of the problem's structure to be able to deduce the formulas of the $n$-period horizon problem. 


\section{The n-period-horizon problem}

The strategy of player $i$ is determined by the Bellman equation,

$$
\begin{aligned}
& V^{i,(n)}\left(k ; \Xi_{0}\right)=\max _{c_{i} \geq 0}\left\{\ln \left(c_{i}\right)+\right. \\
& \left.\quad+\delta \int_{\mathcal{H}} V^{i,(n-1)}\left(B \cdot\left[k-c_{i}-\sum_{j \neq i} C^{j,(n)}\left(k ; \Xi_{0}\right)\right]^{\eta} ; \Xi_{1}(\cdot \mid \eta)\right)\left[\int_{\Theta} \phi(\eta \mid \theta) \xi_{0}^{i}(\theta) \mathrm{d} \theta\right] \mathrm{d} \eta\right\}
\end{aligned}
$$

with $V^{i,(n)}\left(k ; \Xi_{0}\right)$ being of the form,

$$
V^{i,(n)}\left(k ; \Xi_{0}\right)=\left[1+\delta \sum_{t=0}^{n-1} \delta^{t} E_{0}\left(\eta_{t} \mid \xi_{0}^{i}\right)\right] \ln (k)+\kappa^{i,(n)}\left(\Xi_{0}\right),
$$

in which $\kappa^{i,(n)}\left(\Xi_{0}\right)$ is a constant, and

$$
\begin{aligned}
E_{0}\left(\prod_{s=0}^{t} \eta_{s} \mid\right. & \left.\xi_{0}^{i}\right) \equiv \int_{\mathcal{H}} \int_{\Theta} \cdots \int_{\mathcal{H}} \int_{\Theta} \int_{\mathcal{H}} \int_{\Theta} \prod_{s=0}^{t} \eta_{s} \phi\left(\eta_{t} \mid \theta_{t}\right) \xi_{t}^{i}\left(\theta_{t}\right) \mathrm{d} \theta_{t} \mathrm{~d} \eta_{t} \times \\
& \times \phi\left(\eta_{t-1} \mid \theta_{t-1}\right) \xi_{t-1}^{i}\left(\theta_{t-1}\right) \mathrm{d} \theta_{t-1} \mathrm{~d} \eta_{t-1} \times \cdots \times \phi\left(\eta_{0} \mid \theta_{0}\right) \xi_{0}^{i}\left(\theta_{0}\right) \mathrm{d} \theta_{0} \mathrm{~d} \eta_{0} .
\end{aligned}
$$

Moreover, players' strategies are of the form

$$
C^{i,(n)}\left(\Xi_{0}\right)=c^{i,(n)}\left(\Xi_{0}\right) \cdot k, \quad i=1, \ldots, N
$$

in which $\left\{c^{i,(n)}\left(\Xi_{0}\right)\right\}_{i=1}^{N}$ is the unique solution to the linear system,

$$
\left[\begin{array}{cccc}
A^{(n)}\left(\xi_{0}^{1}\right) & 1 & \cdots & 1 \\
1 & A^{(n)}\left(\xi_{0}^{2}\right) & \cdots & 1 \\
\vdots & \vdots & \ddots & \vdots \\
1 & 1 & \cdots & A^{(n)}\left(\xi_{0}^{N}\right)
\end{array}\right] \cdot\left[\begin{array}{c}
c^{1,(n)}\left(\Xi_{0}\right) \\
c^{2,(n)}\left(\Xi_{0}\right) \\
\vdots \\
c^{N,(n)}\left(\Xi_{0}\right)
\end{array}\right]=\left[\begin{array}{c}
1 \\
1 \\
\vdots \\
1
\end{array}\right]
$$

with

$$
A^{(n)}\left(\xi_{0}^{i}\right) \equiv 1+\delta \sum_{t=0}^{n-1} \delta^{t} E_{0}\left(\prod_{s=0}^{t} \eta_{s} \mid \xi_{0}^{i}\right), i \in\{1, \ldots, N\}
$$


To calculate $E_{0}\left(\eta_{t} \mid \xi_{0}^{i}\right)$ we rely on Lemma 1 . From equation (24) of Lemma 1, after setting $h(\eta)=\eta$, the identity function, we obtain,

$$
E_{0}\left(\prod_{s=0}^{t} \eta_{s} \mid \xi_{0}^{i}\right)=\int_{\Theta}\left[\int_{\mathcal{H}} \eta \phi(\eta \mid \theta) \mathrm{d} \eta\right]^{t+1} \xi_{0}^{i}(\theta) \mathrm{d} \theta
$$

and from (6) it is,

$$
E_{0}\left(\prod_{s=0}^{t} \eta_{s} \mid \xi_{0}^{i}\right)=\int_{\Theta}[\mu(\theta)]^{t+1} \xi_{0}^{i}(\theta) \mathrm{d} \theta
$$

Substituting (49) into (46) we obtain,

$$
V^{i,(n)}\left(k ; \Xi_{0}\right)=\left[1+\delta \int_{\Theta} \sum_{t=0}^{n-1} \delta^{t}[\mu(\theta)]^{t+1} \xi_{0}^{i}(\theta) \mathrm{d} \theta\right] \ln (k)+\kappa^{i,(n)}\left(\Xi_{0}\right) .
$$

\section{The infinite-horizon problem}

Notice that since $\mathcal{H} \subseteq(0,1), \mu(\theta) \in(0,1)$ for all $\theta \in \Theta$. After taking the limit when $n \rightarrow \infty,(50)$ gives,

$$
V^{i,(\infty)}\left(k ; \Xi_{0}\right)=V^{i}\left(k ; \Xi_{0}\right)=\left[1+\delta \int_{\Theta} \sum_{t=0}^{\infty} \delta^{t}[\mu(\theta)]^{t+1} \xi_{0}^{i}(\theta) \mathrm{d} \theta\right] \ln (k)+\kappa^{i,(\infty)}\left(\Xi_{0}\right),
$$

so, $\mu(\theta) \in(0,1)$ for all $\theta \in \Theta$ guarantees that $V^{i}(k ; \Xi)$ is well-defined. Since $\xi_{0}^{i}$ is a density function, $\int_{\Theta} \xi_{0}^{i}(\theta) \mathrm{d} \theta=1$, which leads to,

$$
V^{i}(k ; \Xi)=\int_{\Theta} \frac{1}{1-\delta \mu(\theta)} \xi^{i}(\theta) \mathrm{d} \theta \ln (k)+\kappa^{i,(\infty)}(\Xi) .
$$

(Subscript " 0 " of $\Xi_{0}$ has appeared in order to remind that $\Xi_{0}$ denotes prior beliefs in period 0 . In the infinite-horizon setup this timing does not matter any more, so subscript "0" can be dropped. So, we drop it throughout the rest of the proof.) Moreover, the solution is again of the multiplicatively separable form

$$
C^{i,(\infty)}(k ; \Xi)=C^{i}(k ; \Xi)=c^{i}(\Xi) \cdot k
$$


and (48) is generalized to,

$$
\mathbf{A} \cdot\left[\begin{array}{c}
c^{1}(\Xi) \\
c^{2}(\Xi) \\
\vdots \\
c^{N}(\Xi)
\end{array}\right]=\left[\begin{array}{c}
1 \\
1 \\
\vdots \\
1
\end{array}\right]
$$

in which

$$
\mathbf{A} \equiv\left[\begin{array}{cccc}
\int_{\Theta} \frac{1}{1-\delta \mu(\theta)} \xi^{1}(\theta) \mathrm{d} \theta & 1 & \cdots & 1 \\
1 & \int_{\Theta} \frac{1}{1-\delta \mu(\theta)} \xi^{2}(\theta) \mathrm{d} \theta & \cdots & 1 \\
\vdots & \vdots & \ddots & \vdots \\
1 & 1 & \cdots & \int_{\Theta} \frac{1}{1-\delta \mu(\theta)} \xi^{N}(\theta) \mathrm{d} \theta
\end{array}\right]
$$

Since $\mu(\theta) \in(0,1)$ for all $\theta \in \Theta$,

$$
\int_{\Theta} \frac{1}{1-\delta \mu(\theta)} \xi^{i}(\theta) d \theta>1, \quad i \in\{1, . ., N\}
$$

Inequality (52) guarantees that Lemma 3 applies, which implies that the unique solution to (51) is given by equation (9).

Regarding the transversality condition given by (3), notice that according to (4) and (9),

$$
\frac{\partial h^{i}\left(k_{t}^{*}, k_{t+1}^{*} ; \Xi_{t}\right)}{\partial k_{t}} k_{t}=\frac{1-\sum_{j \neq i} c^{j}\left(\Xi_{t}\right)}{c^{i}\left(\Xi_{t}\right)} .
$$

Combining the expression on the left-hand side of (3) with (53) gives,

$$
\lim _{t \rightarrow \infty} \delta^{t} E_{t}\left[\frac{\partial h^{i}\left(k_{t}^{*}, k_{t+1}^{*} ; \Xi_{t}\right)}{\partial k_{t}} k_{t}^{*}\right]=\lim _{t \rightarrow \infty} \delta^{t} \frac{1-\sum_{j \neq i} c^{j}\left(\Xi_{t}\right)}{c^{i}\left(\Xi_{t}\right)}=0,
$$

proving that the transversality condition given by (3) indeed holds.

In order to guarantee that $V^{i}(k ; \Xi)$ is well-defined, it remains to verify condition (11), which can prove important for identifying conditions on $\phi(\cdot \mid \eta)$ and $\Xi_{0}$ that guarantee the 
boundedness of $\kappa^{i,(\infty)}\left(\Xi_{0}\right)$ in applications using specific functional forms. ${ }^{19}$ Specifically, let's take a guess on the functional form of the value function of player $i \in\{1, \ldots, N\}$,

$$
V^{i}(k ; \Xi)=\kappa^{i}(\Xi)+\left[\int_{\Theta} f(\theta) \xi^{i}(\theta) \mathrm{d} \theta\right] \cdot \ln (k)
$$

in which $\kappa^{i}(\Xi)=\kappa^{i,(\infty)}\left(\Xi_{0}\right)$ is an unknown functional and $f$ is given by

$$
f(\theta)=\frac{1}{1-\delta \mu(\theta)},
$$

which is the same for all players $i \in\{1, \ldots, N\}$, since it is independent from specific beliefs $\xi^{i}$. Substituting equation (54) into the Bellman equation (5) gives,

$$
\begin{aligned}
& \kappa^{i}(\Xi)+\left[\int_{\Theta} f(\theta) \xi^{i}(\theta) \mathrm{d} \theta\right] \cdot \ln (k)=\max _{c_{i} \geq 0}\left\{\ln \left(c_{i}\right)+\delta \int_{\mathcal{H}} \kappa^{i}(\Xi(\cdot \mid \eta))\left[\int_{\Theta} \phi(\eta \mid \theta) \xi^{i}(\theta) \mathrm{d} \theta\right] \mathrm{d} \eta\right. \\
& \left.+\delta \ln (B)+\delta \int_{\mathcal{H}} \eta \ln \left[k-c_{i}-\sum_{j \neq i} C^{j}(k ; \Xi)\right] \int_{\Theta} \frac{f(\theta) \phi(\eta \mid \theta) \xi^{i}(\theta)}{\int_{\Theta} \phi(\eta \mid x) \xi^{i}(x) \mathrm{d} x} \mathrm{~d} \theta\left[\int_{\Theta} \phi(\eta \mid \theta) \xi^{i}(\theta) \mathrm{d} \theta\right] \mathrm{d} \eta\right\}
\end{aligned}
$$

which becomes,

$$
\begin{aligned}
\kappa^{i}(\Xi)+ & {\left[\int_{\Theta} f(\theta) \xi^{i}(\theta) \mathrm{d} \theta\right] \cdot \ln (k)=\max _{c_{i} \geq 0}\left\{\ln \left(c_{i}\right)+\delta \int_{\mathcal{H}} \kappa^{i}(\Xi(\cdot \mid \eta))\left[\int_{\Theta} \phi(\eta \mid \theta) \xi^{i}(\theta) \mathrm{d} \theta\right] \mathrm{d} \eta\right.} \\
& \left.+\delta \ln (B)+\delta \int_{\Theta}\left[\int_{\mathcal{H}} \eta \phi(\eta \mid \theta) \mathrm{d} \eta\right] f(\theta) \xi^{i}(\theta) \mathrm{d} \theta \cdot \ln \left[k-c_{i}-\sum_{j \neq i} C^{j}(k ; \Xi)\right]\right\} \cdot(56)
\end{aligned}
$$

First-order conditions based on (56) give,

$$
\frac{1}{c_{i}}=\frac{\delta \int_{\Theta} \mu(\theta) f(\theta) \xi^{i}(\theta) \mathrm{d} \theta}{k-c_{i}-\sum_{j \neq i} C^{j}(k ; \Xi)},
$$

so,

$$
\left[1+\delta \int_{\Theta} \mu(\theta) f(\theta) \xi^{i}(\theta) \mathrm{d} \theta\right] c_{i}+\sum_{j \neq i} C^{j}(k ; \Xi)=k,
$$

$\overline{19} \mathrm{I}$ am indebted to an anonymous referee and the Editor for motivating me to provide the argument that leads to the condition given by (11). 
and if we make the additional guess that the strategies of all other players are of the multiplicatively-separable form $C^{j}(k ; \Xi)=c^{j}(\Xi) \cdot k$, then (57) reconfirms that player $i$ is also of multiplicatively-separable form, namely,

$$
c_{i}=\frac{1-\sum_{j \neq i} c^{j}(\Xi)}{1+\delta \int_{\Theta} \mu(\theta) f(\theta) \xi^{i}(\theta) \mathrm{d} \theta} \cdot k .
$$

Substituting (58) into (56) we identify terms that are multiplicatively-separable expressions of $\ln (k)$ and terms that do not depend on the state variable $k$. Starting from the terms that do not depend on $k$, and which do not affect optimization, these should satisfy the recursion,

$$
\begin{gathered}
\kappa^{i}(\Xi)=\ln \left[\frac{1-\sum_{j \neq i} c^{j}(\Xi)}{1+\delta \int_{\Theta} \mu(\theta) f(\theta) \xi^{i}(\theta) \mathrm{d} \theta}\right]+\delta \int_{\mathcal{H}} \kappa^{i}(\Xi(\cdot \mid \eta))\left[\int_{\Theta} \phi(\eta \mid \theta) \xi^{i}(\theta) \mathrm{d} \theta\right] \mathrm{d} \eta \\
+\delta \ln (B)+\delta \int_{\Theta} \mu(\theta) f(\theta) \xi^{i}(\theta) \mathrm{d} \theta \cdot \ln \left\{\frac{\left[1-\sum_{j \neq i} c^{j}(\Xi)\right] \delta \int_{\Theta} \mu(\theta) f(\theta) \xi^{i}(\theta) \mathrm{d} \theta}{1+\delta \int_{\Theta} \mu(\theta) f(\theta) \xi^{i}(\theta) \mathrm{d} \theta}\right\} \cdot
\end{gathered}
$$

Isolating all terms that are multiplicatively-separable expressions of $\ln (k)$ these must satisfy,

$$
\left[\int_{\Theta} f(\theta) \xi^{i}(\theta) \mathrm{d} \theta\right] \cdot \ln (k)=\ln (k)+\delta\left[\int_{\Theta} \mu(\theta) f(\theta) \xi^{i}(\theta) \mathrm{d} \theta\right] \cdot \ln (k)
$$

which is an expression that complies with function $f(\theta)$, given by (55), verifying the validity of (10). Substituting the expression given by (9) into (59), verifies equations (11) and (12), proving the proposition. 


\section{REFERENCES}

Antoniadou, E., C. Koulovatianos, and L. J. Mirman (2013): Strategic Exploitation of a Common-Property Resource under Uncertainty, Journal of Environmental Economics and Management, 65, 28-39.

Agbo, M. (2013): Strategic exploitation with learning and heterogeneous beliefs, SSRN working paper, No 1973793.

Amir, R. (1996): Continuous Stochastic Games of Capital Accumulation with Convex Transitions, Games and Economic Behavior, 15, 111-131.

Blume, L. and D. Easley (1993): Rational Expectations and Rational Learning, Economics Working Paper Archive (EconWPA), Series: Game Theory and Information, No. 9307003, http://129.3.20.41/eps/game/papers/9307/9307003.pdf

Cogley, T. and Sargent, T. (2008): Anticipated Utility and Rational Expectations as Approximations of Bayesian Decision Making, International Economic Review, 49, $185-221$.

Dutta, P. K., and R. K. Sundaram (1992), Markovian Equilibrium in a Class of Stochastic Games: Existence Theorems for Discounted and Undiscounted Models, Econ. Theory 2, 197-214.

Gelman, A., Carlin, J. B., Stern, H. S., and Rubin, D. B. (2004), "Bayesian Data Analysis," 2nd Edition, London: Chapman \& Hall.

Guidolin, M. and Timmermann, A. (2007): Properties of equilibrium asset prices under alternative learning schemes, Journal of Economic Dynamics and Control, 31, 161-217.

Harville, D. A. (1997): Matrix Algebra From a Statistician's Perspective. SpringerVerlag.

Jackson, M. O. and B. W. Rogers (2007): "Relating Network Structure to Diffusion Properties through Stochastic Dominance," The B.E. Journal of Theoretical Economics, Vol. 7, Iss. 1 (Advances), Article 6. Available at: http://www.bepress.com/bejte/vol7/iss1/art6

Kalai, E. and E. Lehrer (1993): Rational Learning Leads to Nash Equilibrium, Econometrica, 61, 1019-1045.

Koulovatianos, C. (2010): "A Paradox of Environmental Awareness Campaigns", University Library of Munich, MPRA Paper 27260.

Koulovatianos, C., Mirman, L. J. and Santugini, M. (2009): Optimal growth and uncertainty: Learning, Journal of Economic Theory, 144, 280-295. 
Koulovatianos, C. and V. Wieland (2011): Asset Pricing under Rational Learning about Rare Disasters. Centre for Economic Policy Research (CEPR) Discussion Paper No. 8514 .

Levhari, D., and L. J. Mirman (1980): "The Great Fish War: an Example using a Dynamic Cournot-Nash Solution," The Bell Journal of Economics, 11, 322-334.

Lomborg, B. (2001): The Skeptical Environmentalist: Measuring the Real State of the World, Cambridge, UK: Cambridge University Press.

Long, N. V. (2011): Dynamic Games in the Economics of Natural Resources: A Survey. Dynamic Games and Applications, 1, 115-148.

Mawhin, J. (2009): Bounded Solutions: differential vs differene equations, Seventh Mississippi State - UAB Conference on Differential Equations and Computational Simulations, Electronic Journal of Differential Equations, Conf. 17 (2009), pp. 159-170. ISSN: 1072-6691. URL: http://ejde.math.txstate.edu or http://ejde.math.unt.edu

Mirman, L.J, and M. Santugini (2013): "Learning and Technological Progress in Dynamic Games", in Press, forthcoming in Dynamic Games and Applications.

Rörsch, A., T. Frello, R. Soper and A. De Lange (2005): "On the opposition against the book The Skeptical Environmentalist by B. Lomborg," Journal of Information Ethics $14,16-28$.

Sundaram, R. (1989), Perfect equilibrium in a class of symmetric games, J. Econ. Theory 47, 153-177. 


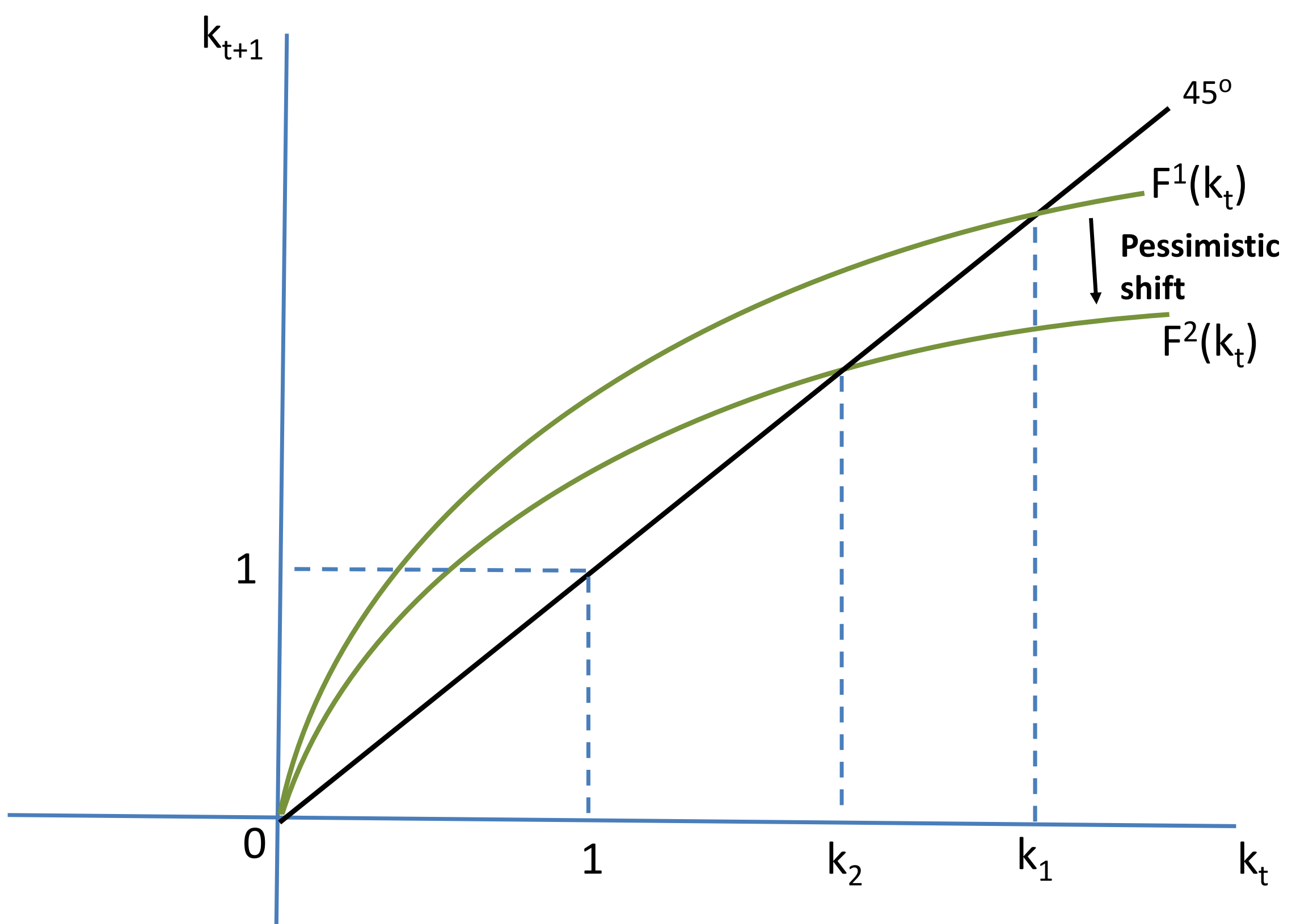

Figure 1 Actual dynamics of $k$ 


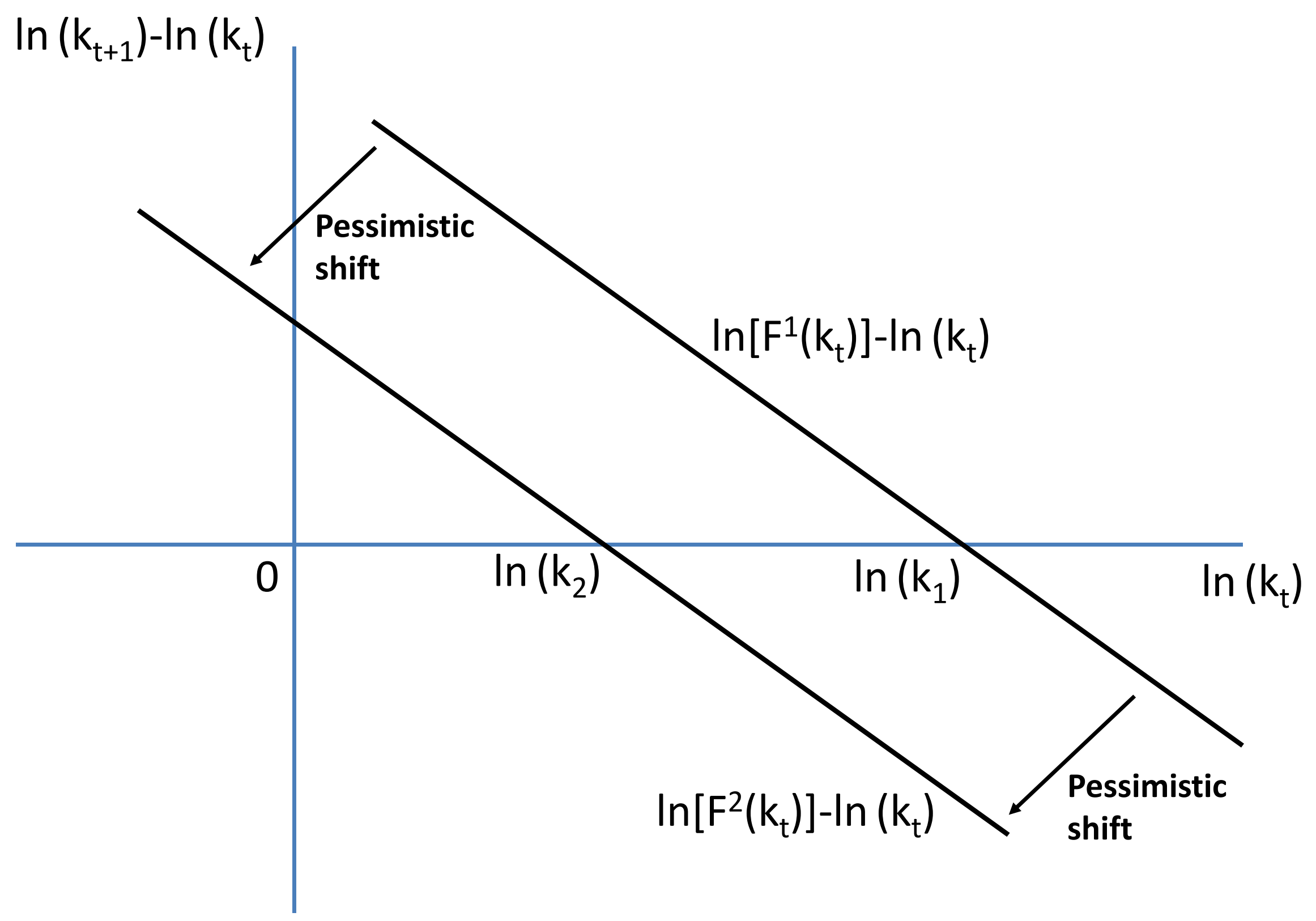

Figure 2 Actual growth rates of $k$ 


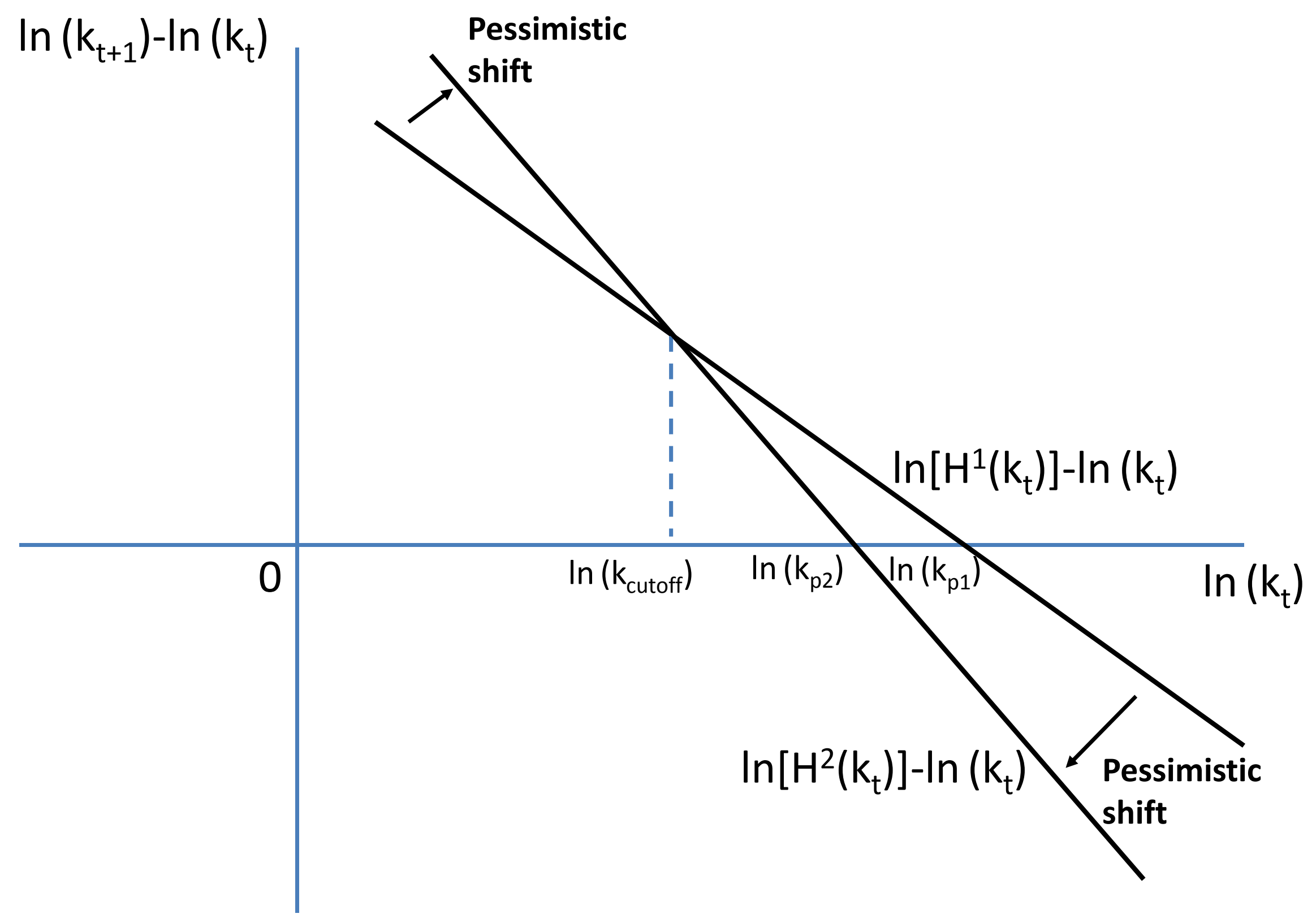

Figure 3 Perceived growth rates of $k$ 


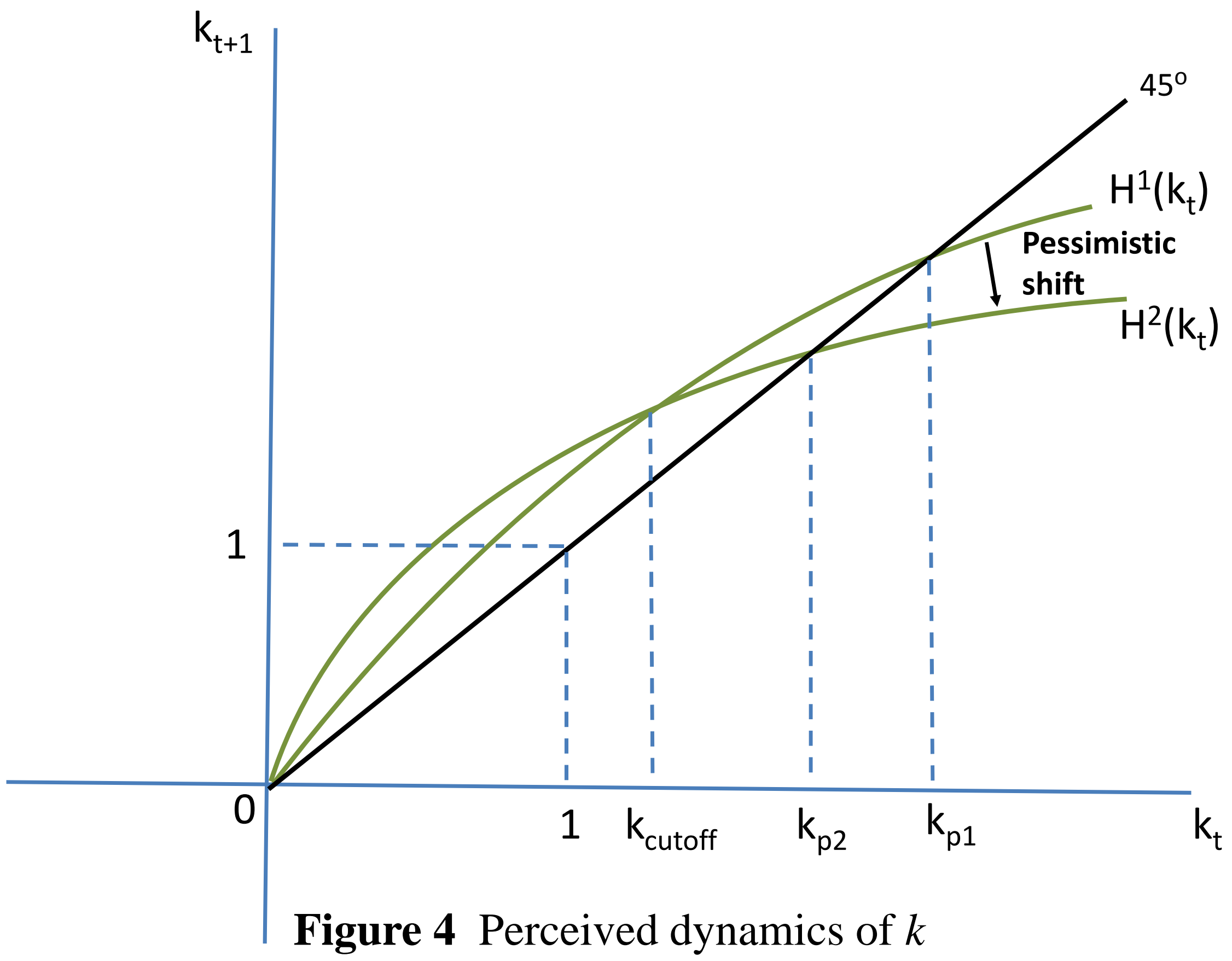

